2018-11

\title{
Wave-structure interaction in hybrid wave farms
}

\author{
Zheng, Siming
}

http://hdl.handle.net/10026.1/13737

10.1016/j.jfluidstructs.2018.09.012

Journal of Fluids and Structures

Elsevier

All content in PEARL is protected by copyright law. Author manuscripts are made available in accordance with publisher policies. Please cite only the published version using the details provided on the item record or document. In the absence of an open licence (e.g. Creative Commons), permissions for further reuse of content should be sought from the publisher or author. 
1

2 Wave-structure interaction in hybrid wave farms

Title:

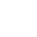

\section{.}

\section{Author names and affiliations:}

Siming Zheng, ${ }^{\mathrm{a}, \mathrm{b}}$, Yongliang Zhang ${ }^{\mathrm{b}}$, Gregorio Iglesias ${ }^{\mathrm{a}}$

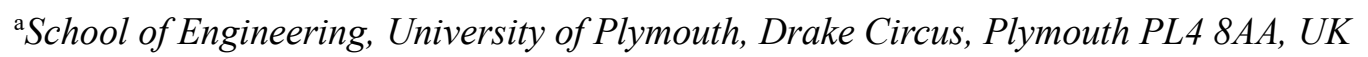

btate Key Laboratory of Hydroscience and Engineering, Tsinghua University, Beijing 100084, China

\section{Email address of each author:}

Siming Zheng: siming.zheng@plymouth.ac.uk/ zhengsm@tsinghua.edu.cn

Yongliang Zhang: yongliangzhang@tsinghua.edu.cn

Gregorio Iglesias: gregorio.iglesias@plymouth.ac.uk

\section{Corresponding author:}

Gregorio Iglesias

Tel: +441752586131

E-mail: address:gregorio.iglesias@plymouth.ac.uk

Received 3 May 2018,

Revised 14 August 2018,

Accepted 25 September 2018,

Available online 11 October 2018.

https://doi.org/10.1016/j.jfluidstructs.2018.09.012 


\begin{abstract}
Hybrid wave farms, consisting of different types of Wave Energy Converters (WECs), have rarely been investigated so far. In this work we propose a hybrid wave farm consisting of Oscillating Water Columns (OWCs) and point-absorbers (PAs), and develop a semi-analytical model of the interaction between this hybrid wave farm and the incident wave field. The OWCs and PAs are modelled as truncated cylinders with and without moonpools, respectively, each with its own outer radius, inner radius, draft and mass. The hydrodynamic model solves the wave diffraction and radiation problems using linear potential flow theory and the methods of separation of variables and eigen-function matching. The independent oscillations of each WEC in any degree of freedom, including both translating and rotating modes, together with the pressure fluctuations of the air inside each OWC chamber, are all accounted for. The model is successfully validated based on numerical data, and thereupon applied to two configurations of the hybrid wave farm. We find that the excitation volume flux/forces are strongly dependent on the incident wave direction, the spacing between the WECs and, more generally, the configuration of the farm. The hydrodynamic coefficients, especially those of the PAs, are sensitive to the spacing and configuration. Notwithstanding the interest of these results in relation to the selection of the optimum configuration and WEC spacing of the hybrid wave farm for specific locations (with specific prevailing wave directions), the interest of this work lies in the semi-analytical model itself, which is found to be efficient in modelling the interaction of the hybrid wave farm and the wave field, and can be used in future wave farm projects.
\end{abstract}

Keywords: Wave energy, Oscillating water column, Point-absorber, Wave excitation volume flux/forces, Hydrodynamic coefficients, semi-analytical model.

\title{
1. Introduction
}

Generated by wind, ocean waves may be regarded as a concentrated form of solar energy. The wave energy resource has been assessed in a number of areas, and the overall conclusion is that the resource is vast, but its spatial and temporal variability is considerable (e.g., Iuppa et al., 2015; López et al., 2015a; Carballo et al., 2015a, 2015b; Khojasteh et al., 2018). The variability may be reduced for the sake of power production by combining wave energy with other renewables (Fusco et al., 2010; Astariz and Iglesias, 2016). In any case, harnessing wave power efficiently still represents a formidable technical challenge, which has inspired inventors and researchers since 1799 (e.g., Ross, 1995; Drew et al., 2009; Falcão, 2010; López et al., 2013; Zheng et al., 2015, 2016a, 2016b; Zheng and Zhang, 2017a, 2017b; Mustapa et al., 2017). This challenge is being tackled at present, with intensive technological developments under way.

The wave power that can be absorbed by a single Wave Energy Converter (WEC) is naturally limited. If wave energy is to provide a significant contribution to the energy mix, wave farms, i.e. arrays of WECs, must be deployed (Falcão, 2010). Unlike a single WEC working in isolation, the evaluation of the performance of a wave farm is all the more complex because of the hydrodynamic interaction between the WECs in the farm, which is significantly influenced by the dimensions and layout of the WECs, the incident wave direction and other parameters.

In this work a wave farm, consisting of different WECs (and hereafter referred to as a hybrid wave farm) is considered. More specifically, Oscillating Water Columns (OWCs) and point-absorbers (PAs) are considered. Both are at the forefront of the technological race in wave 
energy, and are the object of intensive R\&D efforts (e.g., López and Iglesias, 2014; Sheng et al., 2014, 2015a, 2015b; López et al., 2015b; Pereiras et al., 2015; Viviano et al., 2016; Flavià et al., 2017).

Analytical methods are generally efficient for providing a quick and accurate performance estimation of a wave farm consisting of WECs with simple geometries. Siddorn and Eatock Taylor (2008) gave the semi-analytical solution of wave diffraction and radiation from an array of floating cylinders with a view to an application in the field of wave energy, although neither motion nor power values were calculated. The following year, Garnaud and Mei (2009) presented an analytical study to evaluate power extraction by a compact array of small buoys, with spacings well below the typical wavelength. A circular array was found to be advantageous, with better efficiency over a broad range of frequencies than a single large buoy of equal volume. The semi-analytical method adopted by Siddorn and Eatock Taylor (2008) was later employed by Child and Venugopal (2010) to investigate the effects induced by the spatial configuration of an array of heave PAs, which are modelled by heaving truncated cylinders. More recently, a semi-analytical model for heaving PAs was also presented by Göteman (2017), whereas the PAs in this model can be either truncated cylinders or cylinders with moonpools. The results showed that the total power absorption could be improved if the wave farm consisted of devices of different dimensions. Apart from the study on an array of PAs, hydrodynamic analysis of an array of vertical axisymmetric OWCs, either restrained or freely floating, can also be carried out with semi-analytical method (Konispoliatis and Mavrakos, 2016). Theoretical analysis revealed the wave farm with restrained OWCs performed better in capturing wave power than that with floating OWCs. In addition to either OWCs or PAs, a farm consisting of a periodic array of large flap-type WECs was also analytically investigated by Renzi and Dias (2013a; 2013b) and Sarkar et al. (2014). Unlike a line of heaving buoys, an array of flap-type WECs was found to be capable of exploiting the resonance of the transverse modes, resulting in high capture factor levels (Renzi and Dias, 2013a). The analytical study of five layouts of 13 flap-type WECs revealed that a slightly staggered arrangement achieved better power extraction in random seas than an in-line arrangement (Sarkar et al., 2014a). Besides, Sarker et al. (2014b) developed a mathematical model to address the problem of interaction between a flap-type WEC and a PA. Assuming a reasonably large distance of separation between the two WECs, the interaction between them by the evanescent modes of the disturbed potential were neglected.

Apart from analytical methods, various numerical simulations have also been widely used in the study of wave farms, especially when the WECs have complicated geometries. Babarit (2010) modelled wave farms composed of two either surging or heaving WECs using the Boundary Element Method (BEM). The alteration of the absorbed power due to wave interaction effects was found to decrease with the square root of the spacing between the WECs. Later, Göteman et al. (2014) studied different methods of reducing the power output fluctuations of a wave farm of PAs, which included varying: the number of PAs, the separating distance between units, the incoming wave direction, etc. The influence of the interactions between bodies on the overall annual energy production of larger wave farms (consisting of 9 to 25 PAs) was assessed by Borgarino et al. (2012), and the results showed that constructive and destructive interactions compensated each other over the range of wave conditions considered. Penalba et al. (2017) treated the hydrodynamic interactions as a function of the spacing between the WECs; for arrays with very small spacings, interactions were highly destructive. De Andres et al. (2014) employed a 
BEM-based model as well to analyse the influence of the array layout, WEC separation, number of WECs and wave directionality on the power absorption of wave farms composed of two-body heaving WECs. The BEM was also applied by Wu et al. (2017) to study the power capture performance of an array of solo Duck WECs. A better performance of this farm in capturing wave power was presented for the arrays with Duck WECs of smaller width. To investigate interaction of ocean waves with OWCs, Nader (2013) developed a fully numerical three dimensional (3D) finite-element model (FEM) in the framework of the inviscid potential flow theory. Although FEM is very limited in terms of computational domain size due to the computing resources required for the discretisation of the entire water volume, it was deemed better suited to the consideration of internal air pressure effects of OWC and weakly non-linear effects. A FEM-based software was later used by Renzi et al. (2014) to investigate the dynamics of a wave farm made by flap-type WECs in the nearshore, showing that the excitation torque could be maximized when the incident wave acts simultaneously and non-symmetric layouts could be detrimental to the array efficiency. More recently, a numerical wave tank based on 3D incompressible Reynolds-Averaged Navier-Stokes (RANS) equations with consideration of viscous, turbulent and non-linear effects was developed by Devolder et al. (2018) using the computational fluid dynamics (CFD) toolbox OpenFOAM to simulate the wave farm of heaving PAs. Numerical results of heave motion, surge excitation force and surface elevations of wave field agreed well with experimental ones. The far-field effects of wave farms are of interest too, not least in connection with the potential environmental impacts, and have been studied by means of numerical wave models such as SWAN and MIKE 21 (Venugopal and Smith, 2007; Carballo and Iglesias, 2013; Abanades et al., 2014; Iglesias and Carballo, 2014; Astariz and Iglesias, 2015a, 2015b; Astariz et al., 2015; Abanades et al., 2015; López-Ruiz et al., 2018).

In addition, experimental methods have been applied to investigate the performance of wave farms (e.g., Thomas et al., 2008; Weller et al., 2010; Haller et al., 2011; Rahm et al., 2012). Moreover, to improve the power extraction of wave farms, some researchers have focused on the control strategies of WEC arrays, e.g., Folley and Whittaker (2009), Cruz et al. (2010), De Backer et al. (2010), Bacelli and Ringwood (2013).

To the authors' knowledge, most of the previous research works, whether analytical, numerical or experimental, dealt with wave farms consisting of a single type of WEC (and mostly, also a single geometry). In this paper, the authors propose a hybrid wave farm, which is composed of both OWCs and PAs. The hydrodynamic study is the first step toward evaluating the motion/pressure response and performance of a hybrid wave farm in capturing wave power from ocean waves. In this work we develop a semi-analytical model for a hybrid wave farm consisting of any number of OWCs/PAs. The semi-analytical model is validated by comparing the results using different analytical approaches against numerical ones and is further adopted to evaluate effect of wave incident direction and spacing distance between WECs on hydrodynamic performance of the farm with two different deployment configurations.

The rest of this paper is organized as follows. Section 2 presents the governing equations and boundary conditions of both wave diffraction and radiation problems. Diffracted and radiated potentials are expressed and solved in Section 3. Expressions of wave excitation volume flux/forces and hydrodynamic coefficients are given in Section 4. The semi-analytical model is validated in Section 5 and then used to study the hydrodynamic performance of hybrid wave farms in Section 6. Conclusions are summarized in Section 7. 


\section{Mathematical model}

To study the hydrodynamic characteristics of the hybrid wave farm, we consider $M$ OWCs and $N$ PAs arbitrarily deployed on the free surface of a layer of liquid of finite depth $h$ moving independently in the hybrid array (Fig. 1). For convenience, the index $j(j=1,2, \ldots, M+N)$ is used to represent both OWC and PA. For $j \leqslant M$, Float $j$ represents OWC $j$, otherwise, Float $j$ represents PA $j-M$. The submerged depth, the outer radius and the inner radius of Float $j$ are denoted by $d_{j}$, $R_{j}$,and $R_{\mathrm{i}, j}$, respectively. For $j>M$, Float $j$ represents a PA, resulting in a vanishing $R_{\mathrm{i}, j}$.

A general Cartesian coordinate system Oxyz is adopted, with the $O x y$ plane at the location of the mean water surface, and the $O z$ axis pointing upward. The origin point of coordinate $O$ and the direction of the $O x$ axis may be specified arbitrarily in the mean water surface. The OWCs and PAs are subjected to a monochromatic incident wave train of small amplitude $A$ and frequency $\omega$ propagating in the direction $\beta$ relative to the positive $O x$ axis. Additionally, local cylindrical coordinate systems $O_{m} r_{m} \theta_{m}(m=1,2, \ldots, M+N)$ centered on the origin of the OWCs and PAs are defined (see Fig. 1). The rotation center of Float $n$ is $\left(r_{m}=0, z=z_{m}\right)(m=1,2, \ldots, M+N)$, which is used as a reference point to calculate the wave excitation forces and hydrodynamic coefficients in relation with rotary modes. The position of the origin of Float $m$ may be written in terms of Cartesian coordinate system $O x y z$ as $\left(x_{m}, y_{m}\right)$.

For the purpose of analysis, the fluid is divided as follows (see Fig. 1b): (a) fluid domains beneath the OWCs and PAs, which are denoted as Region $m$ (i.e., $R_{\mathrm{i}, m} \leqslant r_{m} \leqslant R_{m},-h \leqslant z \leqslant-d_{m}$ ) and Region $j$ (i.e., $r_{j} \leqslant R_{j},-h \leqslant z \leqslant-d_{j}, j=M+1, M+2, \ldots, M+N$ ), respectively; (b) fluid domains at the interior region of the OWCs and the rest of fluid outside, which are denoted as Region $M+N+m$ (i.e., $r_{m} \leqslant R_{\mathrm{i}, m},-h \leqslant z \leqslant 0$ ) and Region $2 M+N+1$ (i.e., $r_{n} \geqslant R_{n},-h \leqslant z \leqslant 0$ ), respectively.

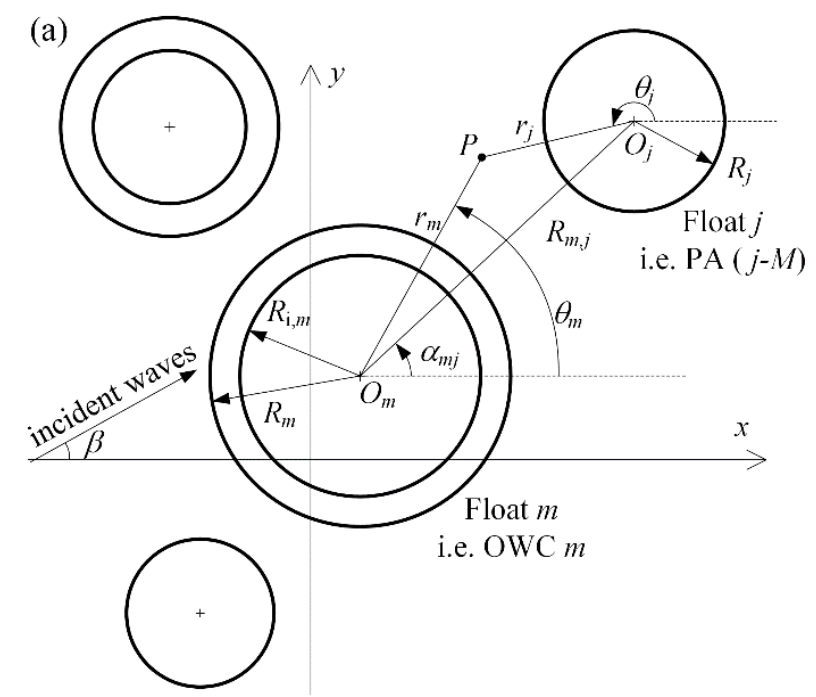




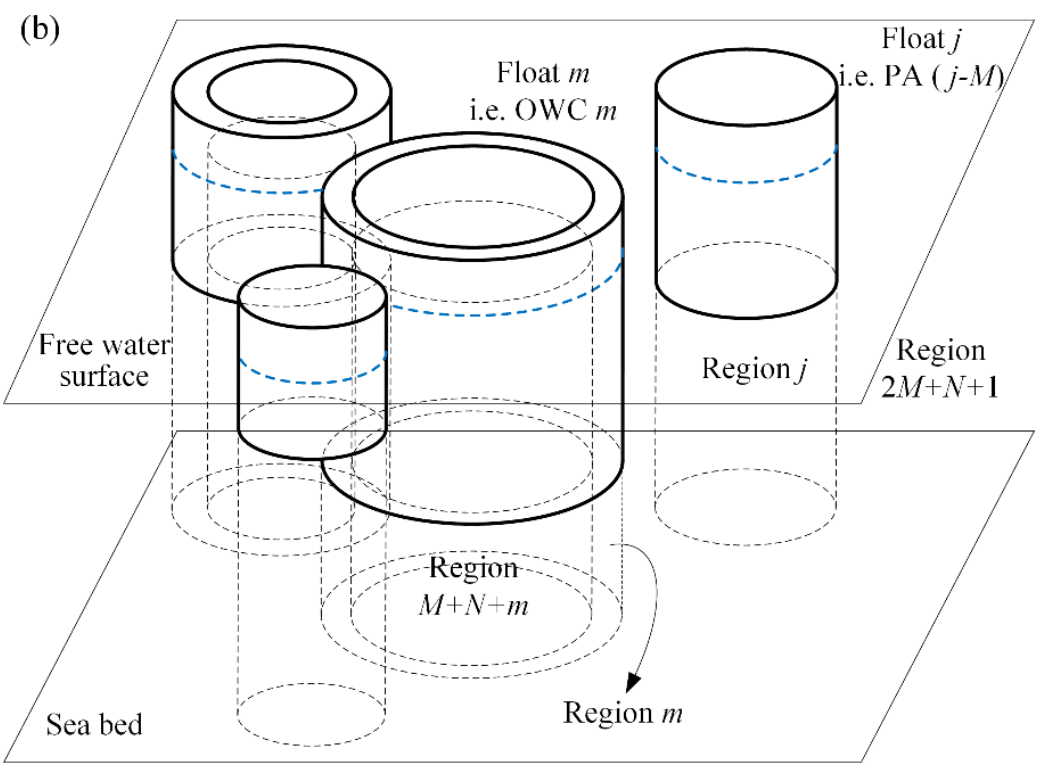

Fig. 1. Definition sketch: a) plan view; b) bird view.

Generally, all OWCs and PAs in the hybrid wave farm are free to oscillate independently in six degrees of freedom. Assuming that the fluid is isotropic, incompressible and inviscid, and that the wave amplitude is small, linear potential flow theory may be adopted to describe the hydrodynamic problem. The total spatial velocity potential $\Phi$ may be decomposed into the incident, diffracted and radiated wave spatial potential as follows:

$$
\Phi=\Phi_{\mathrm{I}}+\Phi_{\mathrm{D}}+\sum_{n=1}^{M+N} \sum_{j=1}^{6} \dot{A}_{n, j} \Phi_{\mathrm{R}}^{n, j}+\sum_{n=1}^{M} p_{n} \Phi_{\mathrm{R}}^{(n)}
$$

where $\Phi_{\mathrm{I}}$ and $\Phi_{\mathrm{D}}$ are the incident and diffracted wave spatial potential, respectively; $\dot{A}_{n, j}$ is the complex velocity amplitude of Float $n$ oscillating in the $j$-th mode $(j=1,2, \ldots, 6$, denotes the oscillation in surge, sway, heave, roll, pitch and yaw, respectively); $\Phi_{\mathrm{R}}^{n, j}$ is the spatial velocity potential due to a unit amplitude velocity oscillation of Float $n$ in $j$-th mode; $p_{n}$ is the complex air pressure amplitude inside the chamber of $\mathrm{OWC} n ; \Phi_{\mathrm{R}}^{(n)}$ is the spatial velocity potential due to unit air pressure oscillation inside the chamber of OWC $n$.

The spatial velocity potential for the undisturbed incident waves with amplitude $A$ and frequency $\omega$ propagating in the direction $\beta$ relative to the positive $O x$ axis may be written as:

$$
\Phi_{\mathrm{I}}=-\frac{\mathrm{i} g A}{\omega} \frac{\cosh \left[k_{0}(z+h)\right]}{\cosh \left(k_{0} h\right)} \mathrm{e}^{\mathrm{i} k_{0}(x \cos \beta+y \sin \beta)}
$$

$$
\Phi_{\mathrm{I}}\left(r_{n}, \theta_{n}, z\right)=-\frac{\mathrm{i} g A}{\omega} \frac{\cosh \left[k_{0}(z+h)\right]}{\cosh \left(k_{0} h\right)} \mathrm{e}^{\mathrm{i} k_{0}\left(x_{n} \cos \beta+y_{n} \sin \beta\right)} \sum_{m=-\infty}^{\infty} \mathrm{i}^{m} \mathrm{e}^{-\mathrm{i} m \beta} J_{m}\left(k_{0} r_{n}\right) \mathrm{e}^{\mathrm{i} m \theta_{n}}
$$

where Eq. (2a) and (2b) are written in the general Cartesian coordinate system $O x y z$ and the local cylindrical coordinate systems $O_{n} r_{n} \theta_{n}$, respectively; $k_{0}$ is the wave number, which satisfies the 
1 dispersion relation $\omega^{2}=g k_{0} \tanh \left(k_{0} h\right) ; g$ is the acceleration of gravity; $\mathrm{i}$ is the imaginary unit; and

$2 J_{m}$ is the Bessel function of order $m$.

The free-surface boundary condition and the body-boundary condition that $\Phi_{\mathrm{D}}, \Phi_{\mathrm{R}}^{j}$, and

$4 \Phi_{\text {OWC }}^{j}$ should satisfy are given as follows:

$5 \quad$ Wave diffracted potentials:

6

7

8

9

13

14

$$
\frac{\partial \Phi_{\mathrm{D}}}{\partial z}-\frac{\omega^{2}}{g} \Phi_{\mathrm{D}}=0, \quad z=0, r_{j} \geq R_{j} \text { and } r_{j}<R_{\mathrm{i}, j}
$$

Wave radiated potentials due to the oscillation of Float $n$ in $i$-th mode:

$$
\frac{\partial \Phi_{\mathrm{R}}^{n, i}}{\partial z}-\frac{\omega^{2}}{g} \Phi_{\mathrm{R}}^{n, i}=0, \quad z=0, r_{j} \geq R_{j} \text { and } r_{j}<R_{\mathrm{i}, j}
$$

$\frac{\partial \Phi_{\mathrm{R}}^{n, i}}{\partial z}=\delta_{n, j}\left(\delta_{3, i}+\delta_{4, i} r_{n} \sin \theta_{n}-\delta_{5, i} r_{n} \cos \theta_{n}\right), \quad z=-d_{j} \quad$ and $\quad R_{\mathrm{i}, j} \leq r_{j} \leq R_{j}$ .

$$
\frac{\partial \Phi_{\mathrm{R}}^{n, i}}{\partial r_{j}}=\delta_{n, j}\left(\delta_{1, i} \cos \theta_{n}+\delta_{2, i} \sin \theta_{n}-\delta_{4, i}\left(z-z_{n}\right) \sin \theta_{n}+\delta_{5, i}\left(z-z_{n}\right) \cos \theta_{n}\right)
$$

$$
-d_{j} \leq z \leq 0, r_{j}=R_{j} \text { and } r_{j}=R_{\mathrm{i}, j}
$$

Wave radiated potential due to the OWC air pressure oscillation inside the chamber of OWC $n$ :

$$
\frac{\partial \Phi_{\mathrm{R}}^{(n)}}{\partial z}-\frac{\omega^{2}}{g} \Phi_{\mathrm{R}}^{(n)}=0, \quad z=0, r_{j} \geq R_{j}
$$

$$
\frac{\partial \Phi_{\mathrm{R}}^{(n)}}{\partial z}-\frac{\omega^{2}}{g} \Phi_{\mathrm{R}}^{(n)}=\delta_{j, n} \frac{\mathrm{i} \omega}{\rho g}, \quad z=0, \quad r_{j}<R_{\mathrm{i}, j}
$$

$$
\frac{\partial \Phi_{\mathrm{R}}^{(n)}}{\partial z}=0, \quad z=-d_{j} \quad \text { and } \quad R_{\mathrm{i}, j} \leq r_{j} \leq R_{j}
$$

$$
\frac{\partial \Phi_{\mathrm{R}}^{(n)}}{\partial r_{j}}=0, \quad-d_{j} \leq z \leq 0, r_{j}=R_{j} \quad \text { and } \quad r_{j}=R_{\mathrm{i}, j}
$$




\section{Semi-analytical solution to diffracted and radiated potentials}

\subsection{Spatial potentials in subdomains}

For clarity, the spatial potentials $\Phi_{\mathrm{D}}, \Phi_{\mathrm{R}}^{n, i}(n=1,2, \ldots, M+N)$, and $\Phi_{\mathrm{R}}^{(n)}(n=1,2, \ldots, M)$ in fluid subdomain Region $j$, can be written in a unified format as $\Phi_{j}^{\chi}$, in which $\chi={ }^{\prime}(0)^{\prime},{ }^{\prime}(n, i)$ ', and ' $(n)$ ' represent the corresponding wave diffracted potential, the radiated potential due to the motion of Float $n$ in $i$-th mode and the radiated potential due to the air pressure oscillation inside Float $n$ 's chamber, respectively. In different regions, applying the variables separation method, the suitable general spatial potentials can be expressed by a complex Fourier series as follows:

1) In Region $j(j=1,2, . ., M+N)$

$$
\Phi_{j}^{\chi}\left(r_{j}, \theta_{j}, z\right)=\Phi_{j, \mathrm{p}}^{\chi}+\sum_{m=-\infty}^{\infty}\left[\frac{D_{m, 0}^{\chi, j}}{2}+\sum_{l=1}^{\infty}\left(A_{m, l}^{\chi, j} \frac{I_{m}\left(\beta_{j, l} r_{j}\right)}{I_{m}\left(\beta_{j, l} R_{j}\right)}+C_{m, l}^{\chi, j} \frac{K_{m}\left(\beta_{j, l} r_{j}\right)}{K_{m}\left(\beta_{j, l} R_{j}\right)}\right) \cos \left[\beta_{j, l}(z+h)\right]\right] \mathrm{e}^{\mathrm{i} m \theta_{j}}
$$

where

$$
D_{m, 0}^{\chi, j}= \begin{cases}A_{m, 0}^{\chi, j}+C_{m, 0}^{\chi, j}\left[1+\ln \left(\frac{r_{j}}{R_{j}}\right)\right], & m=0 \\ A_{m, 0}^{\chi, j}\left(\frac{r_{j}}{R_{j}}\right)^{|m|}+C_{m, 0}^{\chi, j}\left(\frac{r_{j}}{R_{j}}\right)^{-|m|}, \quad m \neq 0\end{cases}
$$

$I_{m}$ is the modified Bessel function of first kind and order $m ; K_{m}$ is the modified Bessel function of second kind and order $m ; A_{m, l}^{\chi, j}$ and $C_{m, l}^{\chi, j}$ are unknown coefficients, in which $C_{m, l}^{\chi, j}$ vanishes for $j>M$, i.e., the region beneath of PA; $\beta_{j, l}$ is the $l$-th eigenvalue in Region $j(j=1,2, \ldots, M+N)$ which is given by

$$
\beta_{j, l}=\frac{l \pi}{h-d_{j}}, l=0,1,2,3, \ldots,
$$

$\Phi_{j, \mathrm{p}}^{\chi}$ is a particular solution, which for $\chi^{\prime}{ }^{\prime}(0)^{\prime}, \Phi_{j, \mathrm{p}}^{\chi}=-\Phi_{\mathrm{I}}$; for $\chi^{\prime}(n)^{\prime}, \Phi_{j, \mathrm{p}}^{\chi}$ vanishes; for $\chi=^{\prime}(n, i)^{\prime}, \Phi_{j, \mathrm{p}}^{\chi}$ can be expressed in coordinate system of Float $j$ as 
1

2

5

6

7

$$
\Phi_{\mathrm{R}, j, \mathrm{p}}^{(n, i)}= \begin{cases}0, \quad i=1,2,6 & \\ \frac{\delta_{n, j}}{4\left(h-d_{j}\right)}\left[2(z+h)^{2}-r_{j}^{2}\right], & i=3 \\ \frac{\delta_{n, j} \sin \theta_{j}}{8\left(h-d_{j}\right)}\left[4 r_{j}(z+h)^{2}-r_{j}^{3}\right], & i=4 \\ \frac{\delta_{n, j} \cos \theta_{j}}{8\left(h-d_{j}\right)}\left[r_{j}^{3}-4 r_{j}(z+h)^{2}\right], & i=5\end{cases}
$$

2) Region $M+N+j(j=1,2, . ., M)$

$$
\Phi_{M+N+j}^{\chi}\left(r_{j}, \theta_{j}, z\right)=\sum_{m=-\infty}^{\infty}\left[\frac{D_{m, 0}^{\chi, M+N+j} J_{m}\left(k_{0} r_{j}\right) Z_{0}(z)}{J_{m}\left(k_{0} R_{\mathrm{i}, j}\right) Z_{0}(0)}+\sum_{l=1}^{\infty} \frac{D_{m, l}^{\chi, M+N+j} I_{m}\left(k_{l} r_{j}\right) Z_{l}(z)}{I_{m}\left(k_{l} R_{\mathrm{i}, j}\right) Z_{l}(0)}\right] \mathrm{e}^{\mathrm{i} m \theta_{j}}+\Phi_{M+N+j, \mathrm{p}}^{\chi}
$$

where $D_{m, l}^{\chi, M+N+j}$ is the coefficient to be solved in Section 3.2; $k_{l}$ is the eigenvalue which is given by (Falnes, 2002)

$$
\begin{gathered}
\omega^{2}=-k_{l} g \tan \left(k_{l} h\right), \quad l=1,2,3, \ldots \\
Z_{0}(z)=N_{0}^{-0.5} \cosh \left[k_{0}(z+h)\right] ; \quad Z_{l}(z)=N_{l}^{-0.5} \cos \left[k_{l}(z+h)\right] ; \\
N_{0}=\frac{1}{2}\left[1+\frac{\sinh \left(2 k_{0} h\right)}{2 k_{0} h}\right] ; \quad N_{l}=\frac{1}{2}\left[1+\frac{\sin \left(2 k_{l} h\right)}{2 k_{l} h}\right]
\end{gathered}
$$

$\Phi_{M+N+j, \mathrm{p}}^{\chi}$ is a particular solution, which for $\chi=^{\prime}(n)^{\prime}, \Phi_{M+N+j, \mathrm{p}}^{\chi}=-\delta_{n, j} \mathrm{i} /(\rho \omega)$; whereas for $\chi={ }^{\prime}(0)^{\prime}$ and ' $(n, i)^{\prime}, \Phi_{M+N+j, \mathrm{p}}^{\chi}$ vanishes.

3) Region $2 M+N+1$

The suitable general spatial potential in Region $2 M+N+1$, can be decomposed into the summation of $M+N$ cylindrical diffracted spatial potentials at the exterior domain as follows:

$$
\Phi_{2 M+N+1}^{\chi}=\sum_{j=1}^{M+N} \Phi_{j}^{\chi, \mathrm{e}}
$$

Diffracted spatial potential $\Phi_{j}^{\chi, \text { e }}$ represents the wave travelling outwards from Float $n$ and can be written in terms of following well-known eigen-function expansion in its own coordinate system:

$$
\Phi_{j}^{\chi, \mathrm{e}}\left(r_{j}, \theta_{j}, z\right)=\sum_{m=-\infty}^{\infty}\left[B_{m, 0}^{\chi, j} \frac{H_{m}\left(k_{0} r_{j}\right)}{H_{m}\left(k_{0} R_{j}\right)} \frac{Z_{0}(z)}{Z_{0}(0)}+\sum_{l=1}^{\infty} B_{m, l}^{\chi, j} \frac{K_{m}\left(k_{l} r_{j}\right)}{K_{m}\left(k_{l} R_{j}\right)} \frac{Z_{l}(z)}{Z_{l}(0)}\right] \mathrm{e}^{\mathrm{i} m \theta_{j}}
$$

where $H_{m}$ is the Hankel function of first kind of order $m ; B_{m, l}^{\chi, n}$ are unknown coefficients to be 
4

5

solved in Section 3.2;

Graf's addition theorem for Bessel functions (Abramowitz and Stegun, 1964) is adopted here, thus $\Phi_{2 M+N+1}^{\chi}$ can be expressed in the polar coordinates $\mathrm{O}_{j} r_{j} \theta_{j} z$ as (Zheng and Zhang, 2015):

$$
\begin{aligned}
& \Phi_{2 M+N+1}^{\chi}\left(r_{j}, \theta_{j}, z\right) \\
& =\sum_{m=-\infty}^{\infty}\left[B_{m, 0}^{\chi, j} \frac{H_{m}\left(k_{0} r_{j}\right)}{H_{m}\left(k_{0} R_{j}\right)} \frac{Z_{0}(z)}{Z_{0}(0)}+\sum_{l=1}^{\infty} B_{m, l}^{\chi, j} \frac{K_{m}\left(k_{l} r_{j}\right)}{K_{m}\left(k_{l} R_{j}\right)} \frac{Z_{l}(z)}{Z_{l}(0)}\right] \mathrm{e}^{\mathrm{i} m \theta_{j}}
\end{aligned}
$$

$$
\begin{aligned}
& +\sum_{\substack{j^{\prime}=1 \\
j^{\prime} \neq j}}^{M+N} \sum_{m=-\infty}^{\infty}\left\{\frac{B_{m, 0}^{\chi, j^{\prime}}}{H_{m}\left(k_{0} R_{j^{\prime}}\right)} \frac{Z_{0}(z)}{Z_{0}(0)} \sum_{m^{\prime}=-\infty}^{\infty}(-1)^{m^{\prime}} H_{m-m^{\prime}}\left(k_{0} R_{j j^{\prime}}\right) J_{m^{\prime}}\left(k_{0} r_{j}\right) \mathrm{e}^{\mathrm{i}\left(m \alpha_{j j^{\prime}}-m^{\prime} \alpha_{j j^{\prime}}\right)} \mathrm{e}^{\mathrm{i} m^{\prime} \theta_{j}}\right. \\
& \left.\quad+\sum_{l=1}^{\infty} \frac{B_{m, l}^{\chi, j^{\prime}}}{K_{m}\left(k_{l} R_{j^{\prime}}\right)} \frac{Z_{l}(z)}{Z_{l}(0)} \sum_{m^{\prime}=-\infty}^{\infty} K_{m-m^{\prime}}\left(k_{l} R_{j j^{\prime}}\right) I_{m^{\prime}}\left(k_{l} r_{j}\right) \mathrm{e}^{\mathrm{i}\left(m \alpha_{j^{\prime}}-m^{\prime} \alpha_{j j^{\prime}}\right)} \mathrm{e}^{\mathrm{i} m^{\prime} \theta_{j}}\right\}
\end{aligned}
$$

$r_{j}<R_{j j^{\prime}}$

\subsection{Method of computation for unknown coefficients}

Expressions of the diffracted and radiated spatial potentials as given in Eqs. (12) (22) in Sections 3.1 satisfy all the boundary conditions as shown in Eqs. (3) (11) given in Section 2, except those on the interfaces of each two adjacent subdomains $r_{j}=R_{j}$ and $r_{j}=R_{\mathrm{i}, j}$. The conditions of continuity for pressure and normal velocity at $r_{j}=R_{j}$ and $r_{j}=R_{\mathrm{i}, j}$ can be used to determine the unknown coefficients in Eqs. (12) (22) for both diffracted and radiated spatial potentials.

The continuity conditions for the spatial potentials are given as follows:

1) Continuity of pressure at the boundary $r_{j}=R_{j}(j=1,2, \ldots M+N)$ :

$$
\Phi_{2 M+N+1}^{\chi}\left(r_{j}, \theta_{j}, z\right)=\Phi_{j}^{\chi}\left(r_{j}, \theta_{j}, z\right), \quad-h<z<-d_{j}, r_{j}=R_{j}
$$

2) Continuity of pressure at the boundary $r_{j}=R_{\mathrm{i}, j}(j=1,2, \ldots M)$ :

$$
\Phi_{M+N+j}^{\chi}\left(r_{j}, \theta_{j}, z\right)=\Phi_{j}^{\chi}\left(r_{j}, \theta_{j}, z\right), \quad-h<z<-d_{j}, r_{j}=R_{\mathrm{i}, j}
$$

3) Continuity of normal velocity at the boundary $r_{j}=R_{j}(j=1,2, \ldots M+N)$ :

For $-h<z<-d_{j}$,

$$
\frac{\partial \Phi_{2 M+N+1}^{\chi}\left(r_{j}, \theta_{j}, z\right)}{\partial r_{j}}=\frac{\partial \Phi_{j}^{\chi}\left(r_{j}, \theta_{j}, z\right)}{\partial r_{j}}
$$

For $-d_{j}<z<0$, 
$1 \frac{\partial \Phi_{2 M+N+1}^{\chi}\left(r_{j}, \theta_{j}, z\right)}{\partial r_{j}}= \begin{cases}-\frac{\partial \Phi_{\mathrm{I}}\left(r_{j}, \theta_{j}, z\right)}{\partial r_{j}}, & \chi={ }^{\prime}(0)^{\prime} \\ \delta_{n, j}\left[\delta_{1, i} \cos \theta_{n}+\delta_{2, i} \sin \theta_{n}\right. & \\ \left.-\delta_{4, i}\left(z-z_{n}\right) \sin \theta_{n}+\delta_{5, i}\left(z-z_{n}\right) \cos \theta_{n}\right], & \chi=\text { ' }^{\prime}(n, i)^{\prime} \\ 0, & \chi='(n)^{\prime}\end{cases}$

2

3

4

5

6
8

4) Continuity of normal velocity at the boundary $r_{j}=R_{\mathrm{i}, j}(j=1,2, \ldots M)$ :

$$
\text { For }-h<z<-d_{j}
$$

$$
\frac{\partial \Phi_{M+N+j}^{\chi}\left(r_{j}, \theta_{j}, z\right)}{\partial r_{j}}=\frac{\partial \Phi_{j}^{\chi}\left(r_{j}, \theta_{j}, z\right)}{\partial r_{j}}
$$

For $-d_{j}<z<0$

$\frac{\partial \Phi_{M+N+j}^{\chi}\left(r_{j}, \theta_{j}, z\right)}{\partial r_{j}}= \begin{cases}-\frac{\partial \Phi_{\mathrm{I}}\left(r_{j}, \theta_{j}, z\right)}{\partial r_{j}}, & \chi=\text { ' }^{\prime}(0)^{\prime} \\ \delta_{n, j}\left[\delta_{1, i} \cos \theta_{n}+\delta_{2, i} \sin \theta_{n}\right. & \\ \left.-\delta_{4, i}\left(z-z_{n}\right) \sin \theta_{n}+\delta_{5, i}\left(z-z_{n}\right) \cos \theta_{n}\right], & \chi=\text { ' }^{\prime}(n, i)^{\prime} \\ 0, & \chi=\text { ' }^{\prime}(n)^{\prime}\end{cases}$

Upon substituting the diffracted and radiated spatial potentials in Eqs. (12) (22) into Eqs. (23) $\sim(26)$, utilizing the orthogonal properties of the functions $\cos (n \theta), \sin (n \theta)$, and $Z_{l}(\mathrm{z})$ (Zheng and Zhang, 2015; 2016), and making some rearrangements, the diffracted spatial potentials and the radiated ones in each subdomain can be obtained by solving a matrix equation, in which the infinite series are truncated by choosing $\left(2 m_{0}+1\right)$ terms $\left(m=-m_{0}, \ldots, 0, \ldots, m_{0}\right)$ for $\mathrm{e}^{\mathrm{i} m \theta}$ functions and $l_{0}+1$ terms $\left(l=0,1,2, \ldots l_{0}\right)$ for $Z_{l}(\mathrm{z})$ and $\cos \left[\beta_{n, l}(z+h)\right]$ functions. The brief derivation and the final complicated formulae for calculation of these unknown coefficients are given in Appendix A.

\section{Wave excitation volume flux/forces and hydrodynamic coefficients}

\subsection{Wave excitation volume flux/forces}

Wave excitation volume flux is the upward flux at the water surface inside the OWC chamber due to the contributions of undisturbed incident wave and the diffracted wave when the dynamic air pressure is zero. Expression of the wave excitation volume flux can be written as $\operatorname{Re}\left[F_{\mathrm{e}}^{(n)} \mathrm{e}^{-\mathrm{i} \omega t}\right]$, where, with utilization of Eq.(2) and Eq.(16), 
1

$$
\begin{aligned}
F_{\mathrm{e}}^{(n)} & =\left.\int_{0}^{2 \pi} \int_{0}^{R_{\mathrm{i}, n}} \frac{\partial\left(\Phi_{\mathrm{I}}+\Phi_{\mathrm{D}}\right)}{\partial z}\right|_{z=0} r_{n} \mathrm{~d} r_{n} \mathrm{~d} \theta_{n}=\left.\frac{\omega^{2}}{g} \int_{0}^{2 \pi} \int_{0}^{R_{\mathrm{i}, n}}\left(\Phi_{\mathrm{I}}+\Phi_{M+N+n}^{(0)}\right)\right|_{z=0} r_{n} \mathrm{~d} r_{n} \mathrm{~d} \theta_{n} \\
& =\frac{2 \pi \omega^{2} R_{\mathrm{i}, n}}{g}\left[-\frac{\mathrm{i} g A}{\omega} \mathrm{e}^{\mathrm{i} k_{0}\left(x_{n} \cos \beta+y_{n} \sin \beta\right)} \frac{J_{1}\left(k_{0} R_{\mathrm{i}, n}\right)}{k_{0}}+\frac{D_{0,0}^{(0), M+N+n} J_{1}\left(k_{0} R_{\mathrm{i}, n}\right)}{k_{0} J_{0}\left(k_{0} R_{\mathrm{i}, n}\right)}+\sum_{l=1}^{\infty} \frac{D_{0, l}^{(0), M+N+n} I_{1}\left(k_{l} R_{\mathrm{i}, n}\right)}{k_{l} I_{0}\left(k_{l} R_{\mathrm{i}, n}\right)}\right]
\end{aligned}
$$

Wave excitation forces are the forces due to the incident wave acting on structures which are stationary. It can be computed from the incident wave potential and the diffracted potential (Finnegan et al., 2013). The generalized wave excitation force on Float $n$ in $i$-th mode is $\operatorname{Re}\left[F_{\mathrm{e}}^{n, i} \mathrm{e}^{-\mathrm{i} \omega t}\right]$, where

$$
F_{\mathrm{e}}^{n, i}=-\mathrm{i} \omega \rho \int_{S_{n}}\left(\Phi_{\mathrm{I}}+\Phi_{\mathrm{D}}\right) n_{i} \mathrm{~d} s,
$$

$S_{n}$ represents the wet surface of Float $n$.

\subsection{Hydrodynamic coefficients}

When the water column inside the chamber or the floats oscillates in the absence of an incident wave, the radiated wave reacts with an upward flux at the water surface inside the OWC chamber, so-called radiation volume flux, and forces on the floats, so-called radiation forces.

The complex amplitudes of radiation volume flux due unit amplitude velocity oscillation of Float $n$ oscillating in $i$-th mode can be respectively written into imaginary and real parts as:

$$
\begin{aligned}
& F_{\mathrm{R}, n, i}^{(j)}=\left.\int_{0}^{2 \pi} \int_{0}^{R_{\mathrm{i}, j}} \frac{\partial \Phi_{j}^{(n, i)}}{\partial z}\right|_{z=0} r_{j} \mathrm{~d} r_{j} \mathrm{~d} \theta_{j}=\frac{\omega^{2}}{g} \int_{0}^{2 \pi} \int_{0}^{R_{\mathrm{i}, j}} \Phi_{j}^{(n, i)} r_{j} \mathrm{~d} r_{j} \mathrm{~d} \theta_{j} \\
& =\frac{2 \pi \omega^{2} R_{\mathrm{i}, j}}{g}\left[\frac{D_{0,0}^{(n, i), M+N+j} J_{1}\left(k_{0} R_{\mathrm{i}, j}\right)}{k_{0} J_{0}\left(k_{0} R_{\mathrm{i}, j}\right)}+\sum_{l=1}^{\infty} \frac{D_{0, l}^{(n, i), M+N+j} I_{1}\left(k_{l} R_{\mathrm{i}, j}\right)}{k_{l} I_{0}\left(k_{l} R_{\mathrm{i}, j}\right)}\right]=\mathrm{i} \omega a_{n, i}^{(j)}-c_{n, i}^{(j)}
\end{aligned}
$$

where $a_{n, i}^{(j)}$ and $c_{n, i}^{(j)}$ are the hydrodynamic coefficients.

Similarly, the complex amplitudes of the radiation force exerted on Float $n$ ' in $i^{\prime}$-th mode due to unit amplitude velocity oscillation of Float $n$ oscillating in $i$-th mode can be respectively written in terms of hydrodynamic coefficients $a_{n, i}^{n^{\prime}, i^{\prime}}$ and $c_{n, i}^{n^{\prime}, i^{\prime}}$ as:

$$
F_{\mathrm{R}, n, i}^{n^{\prime}, i^{\prime}}=-\mathrm{i} \omega \rho \int_{S_{n^{\prime}}} \Phi_{\mathrm{R}}^{n, i} n_{i^{\prime}} \mathrm{d} s=\mathrm{i} \omega a_{n, i}^{n^{\prime}, i^{\prime}}-c_{n, i}^{n^{\prime}, i^{\prime}} .
$$

Similar expressions may be obtained for the complex amplitudes of radiation volume and the complex amplitude of radiation force exerted on $n$ ' in $i$ '-th mode due to unit air pressure oscillation inside the chamber of OWC $n$, and the corresponding hydrodynamic coefficients are denoted as $a_{(n)}^{(j)}, c_{(n)}^{(j)}$, and $a_{(n)}^{n^{\prime}, i^{\prime}}, c_{(n)}^{n^{\prime}, i^{\prime}}$, respectively.

The method for calculating the hydrodynamic coefficients as given in Eqs. (29) (30) is straightforward based on the definitions of radiation volume flux and radiation forces. To distinguish this method from the others proposed below (Section 4.3), it will be referred 
1

henceforth as the "direct method".

\subsection{Hydrodynamic coefficients in terms of wave excitation volume flux/forces}

It is known that there is a Haskind relation between wave diffraction and radiation problems (Falnes, 2002), and therefore the hydrodynamic coefficients can be written in terms of wave excitation volume flux and wave excitation forces as:

$$
c_{(n)}^{(j)}=\frac{k}{8 \pi \rho g v_{\mathrm{g}} A^{2}} \int_{0}^{2 \pi} F_{\mathrm{e}}^{(j)}(\beta) F_{\mathrm{e}}^{(n) *}(\beta) \mathrm{d} \beta,
$$

where ' $*$ ' denotes the complex-conjugate, $v_{\mathrm{g}}$ is the wave group velocity expressed as

$$
v_{\mathrm{g}}=\frac{\omega}{2 k}\left[1+\frac{2 k h}{\sinh (2 k h)}\right] \text {. }
$$

\section{Model validation}

To validate the hydrodynamic analysis model, we consider a hybrid wave farm consisting of four truncated floats, in which two are OWCs and the other two are PAs (Fig. 2). All of these structures are half submerged in the water. Table 1 gives a list of the dimensions of the floats. In addition, the physical parameters such as the water depth $h$ and the sea water density $\rho$ are listed in Table 2 . The reference rotation center of each float is set to $(0,0)$ in their own local cylindrical coordinate systems. 


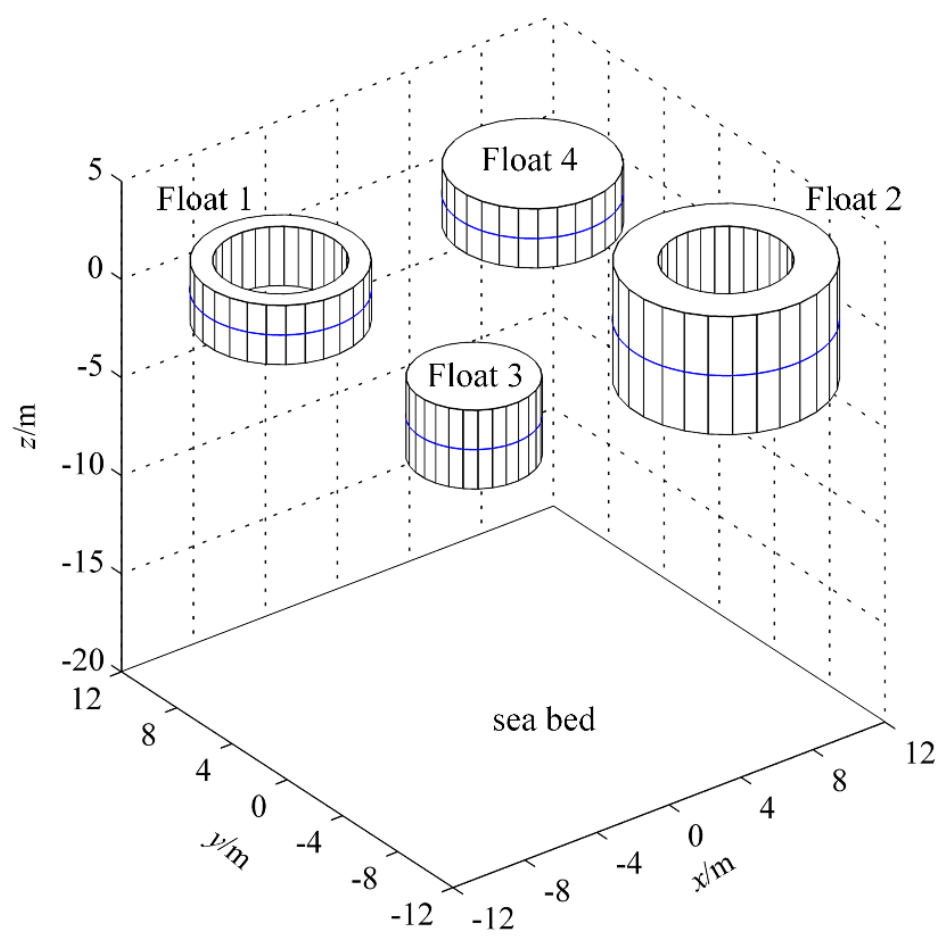

Fig. 2. Top view of the hybrid wave farm consisting of two OWCs and two PAs.

Table 1. Basic parameters of the floats as shown in Fig. 2. (Units: $m$ )

\begin{tabular}{ccccc}
\hline & Float 1 & Float 2 & Float 3 & Float 4 \\
\hline Centre position, $\left(x_{n}, y_{n}\right)$ & $(-7.0,7.0)$ & $(7.0,-7.0)$ & $(-7.0,-7.0)$ & $(7.0,7.0)$ \\
Radius, $R_{n}$ & 4.0 & 5.0 & 3.0 & 4.0 \\
Inner radius, $R_{\mathrm{i}, n}$ & 3.0 & 3.0 & - & - \\
draft, $d_{n}$ & 1.5 & 3.0 & 2.0 & 1.5 \\
\hline
\end{tabular}

Table 2. Physical parameters

\begin{tabular}{cccc}
\hline water depth, $h$ & sea water density, $\rho$ & acceleration of gravity, $g$ & wave incoming direction, $\beta$ \\
\hline $20 \mathrm{~m}$ & $1025 \mathrm{~kg} / \mathrm{m}^{3}$ & $9.81 \mathrm{~m} / \mathrm{s}^{2}$ & $30^{\circ}$ \\
\hline
\end{tabular}

The dimensionless quantities of the wave excitation volume flux/forces and hydrodynamic coefficients are defined by:

$$
\bar{F}_{\mathrm{e}}^{(n)}=\frac{\omega\left|F_{\mathrm{e}}^{(n)}\right|}{g R_{0} A} ; \varphi_{\mathrm{e}}^{(n)}=\arg F_{\mathrm{e}}^{(n)} ; \bar{F}_{\mathrm{e}}^{n, i}=\frac{\left|F_{\mathrm{e}}^{n, i}\right|}{\rho g R_{0}^{j} A} ; \varphi_{\mathrm{e}}^{n, i}=\arg F_{\mathrm{e}}^{n, i},
$$

where $R_{0}$ is a reference length, which is chosen as the average radius of the floats as $R_{0}=4.0 \mathrm{~m}$ for the case as given in Fig. 2 and Table $1 ; j=2$ for $i=1 \sim 3$; whereas $j=3$ for $i=4 \sim 5$.

$$
\bar{a}_{n}^{\left(n^{\prime}\right)}=\frac{\omega^{2} \rho a_{n}^{\left(n^{\prime}\right)}}{R_{0}} ; \bar{c}_{n}^{\left(n^{\prime}\right)}=\frac{\omega \rho c_{n}^{\left(n^{\prime}\right)}}{R_{0}} ; \bar{a}_{n, i}^{n^{\prime}, i^{\prime}}=\frac{a_{n, i}^{n^{\prime}, i^{\prime}}}{\rho R_{0}^{j}} ; \bar{c}_{n, i}^{n^{\prime}, i^{\prime}}=\frac{c_{n, i}^{n^{\prime}, i^{\prime}}}{\omega \rho R_{0}^{j}},
$$

where $j=3$ for $\left(i, i^{\prime}\right)=(1 \sim 3,1 \sim 3) ; j=4$ for $\left(i, i^{\prime}\right)=(1 \sim 3,4 \sim 5)$ and $(4 \sim 5,1 \sim 3)$; whereas $j=5$ for $(i$, $\left.i^{\prime}\right)=(4 \sim 5,4 \sim 5)$. 


$$
\bar{a}_{(n)}^{n^{\prime}, i}=\frac{\omega a_{(n)}^{n^{\prime}, i}}{R_{0}^{j}} ; \bar{c}_{(n)}^{n^{\prime}, i}=\frac{c_{(n)}^{n^{\prime}, i}}{R_{0}^{j}} ; \bar{a}_{n, i}^{\left(n^{\prime}\right)}=\frac{\omega a_{n, i}^{\left(n^{\prime}\right)}}{R_{0}^{j}} ; \bar{c}_{n, i}^{\left(n^{\prime}\right)}=\frac{c_{n, i}^{\left(n^{\prime}\right)}}{R_{0}^{j}},
$$

where $j=2$ for $i=1 \sim 3$; whereas $j=3$ for $i=4 \sim 5$.

A conventional BEM (Boundary Element Method) based commercial code, ANASYS AQWA (ANSYS AQWA, 2011), is adopted to study the wave diffraction and radiation problem from the same case as given in Fig. 2 and Table 1 as a comparison. The CPU time required to achieve the numerical simulation using 8916 wetted elements is about 16 mins for each frequency.

The semi-analytical model described above has been implemented as a house developed computer code in FORTRAN programming language. In our computations for the case as given in Fig. 2 and Table 1, a convergence analysis is carried out to obtain accurate results using the eigen-series analysis. In Figs. 3 and 4, the convergence study of the wave excitation volume flux/forces and hydrodynamic coefficients are presented to illustrate the impact of the angular and vertical truncated cut-offs (i.e., in terms of $m_{0}$ and $l_{0}$ ), respectively.

(a)

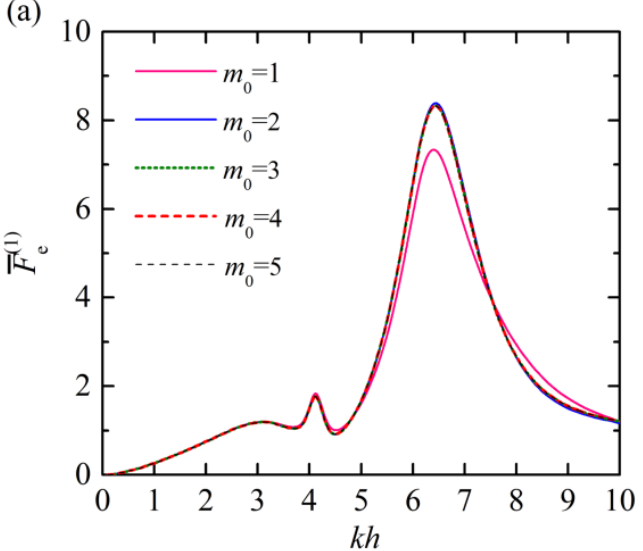

(c)

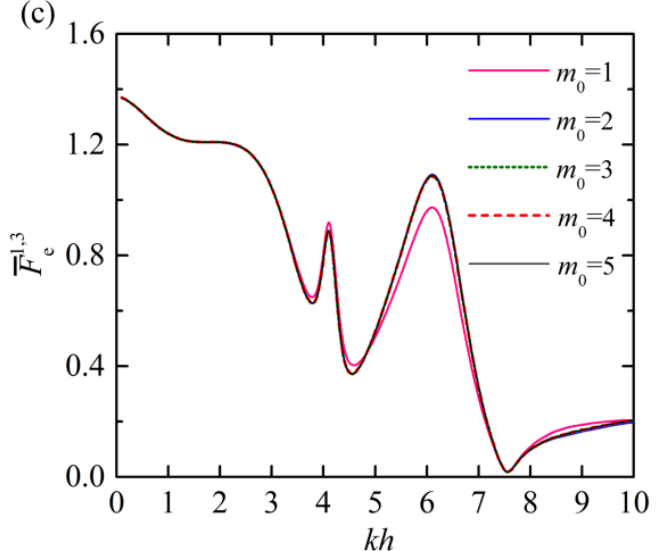

(b)

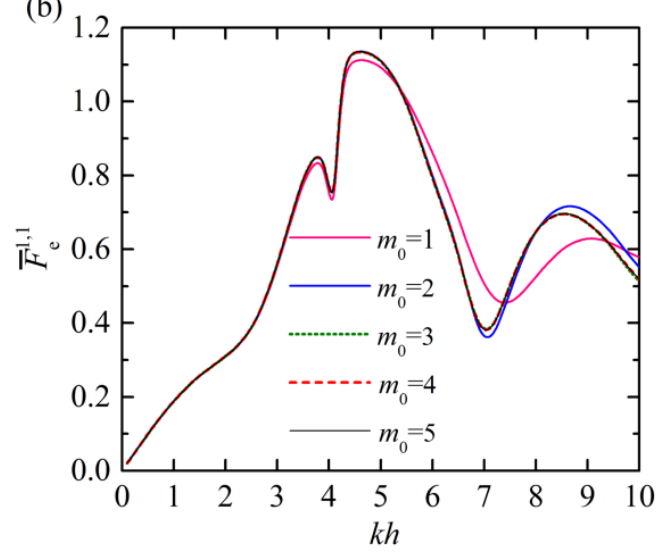

(d) 0

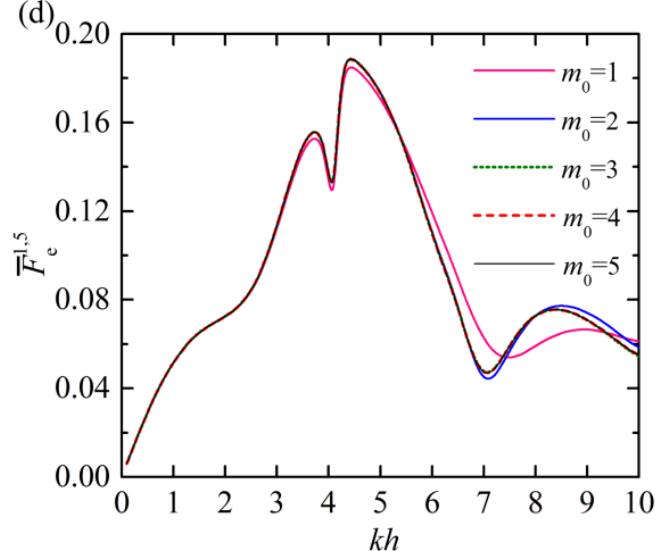

Fig. 3. Impact of the angular cut-offs (i.e., in terms of $m_{0}$ ) on wave excitation volume flux/forces, $l_{0}=30$. (a) $\bar{F}_{\mathrm{e}}^{(1)}$; (b) $\bar{F}_{\mathrm{e}}^{1,1}$; (c) $\bar{F}_{\mathrm{e}}^{1,3} ;$ (d) $\bar{F}_{\mathrm{e}}^{1,5}$. 
(a)

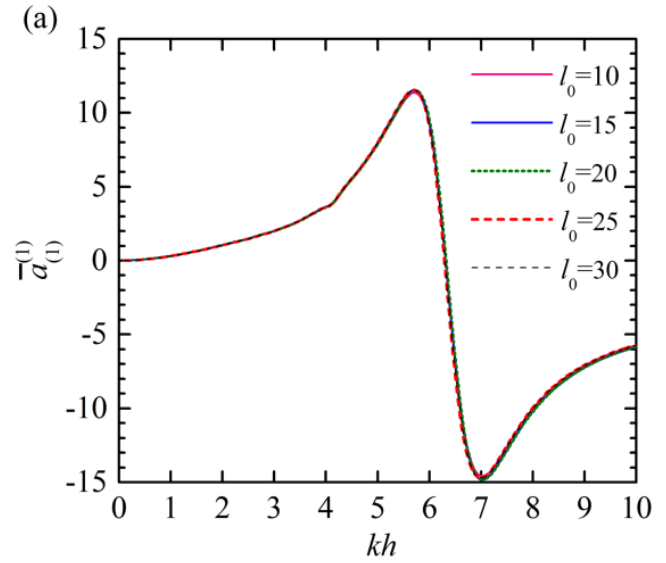

(c)

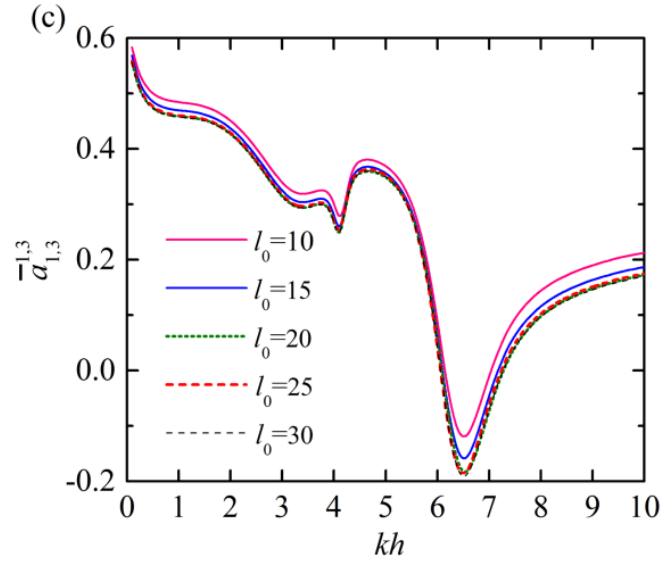

(e)

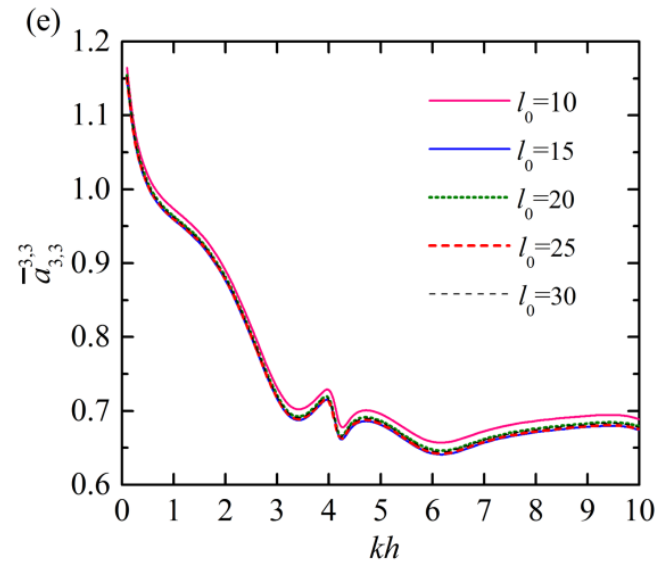

(b)

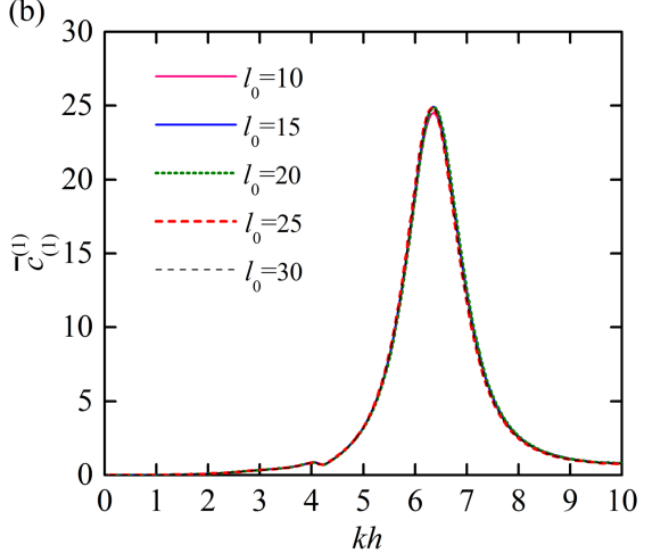

(d)
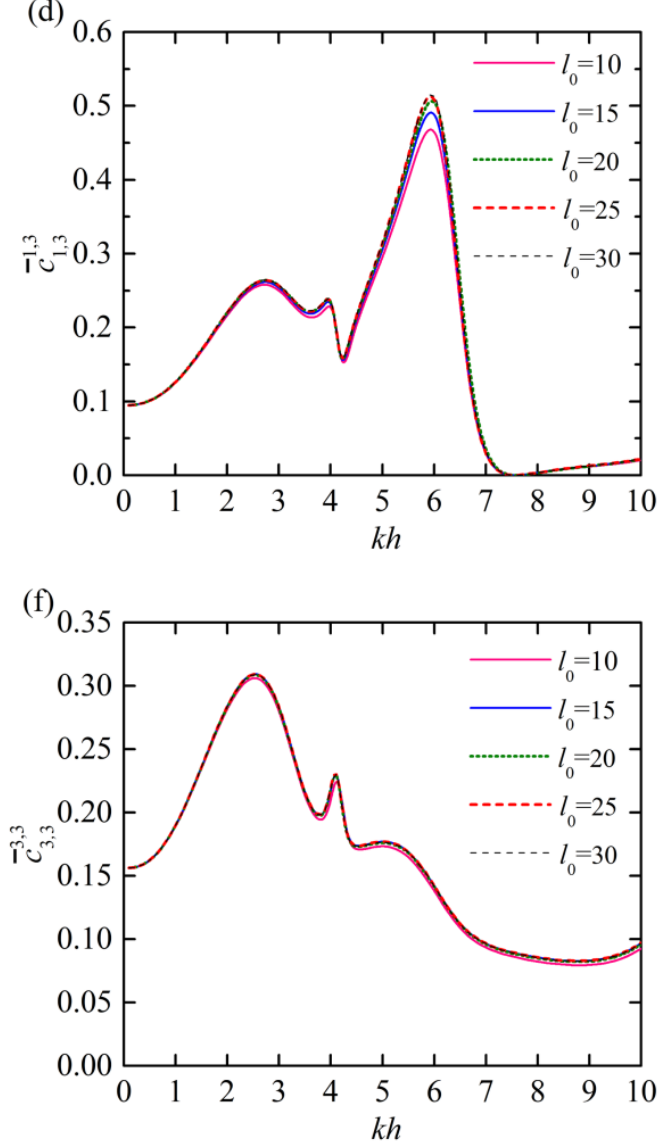

Fig. 4. Impact of the vertical cut-offs (i.e., in terms of $l_{0}$ ) on hydrodynamic coefficients, $m_{0}=5$. (a)

$$
\bar{a}_{(1)}^{(1)} \text {; (b) } \bar{c}_{(1)}^{(1)} \text {; (c) } \bar{a}_{1,3}^{1,3} ; \text { (d) } \bar{c}_{1,3}^{1,3} \text {; (e) } \bar{a}_{3,3}^{3,3} \text {; (f) } \bar{c}_{3,3}^{3,3} .
$$

In order to obtain the converged results, $m_{0} \geqslant 4$ and $l_{0} \geqslant 25$ are suggested as learnt from Figs. 3 and 4 . Hereinafter, $m_{0}=5$ and $l_{0}=30$ are adopted. The CPU time required for solving the diffraction and radiation problem for each wave frequency is merely about 30 seconds by the semi-analytical model, providing a more efficient tool than numerical models.

The semi-analytical and numerical results for wave diffraction and radiation are presented below. 
$1 \quad 5.1$ Wave diffraction

2 The results for wave diffraction problem are plotted in Fig. 5.

3 
(a)

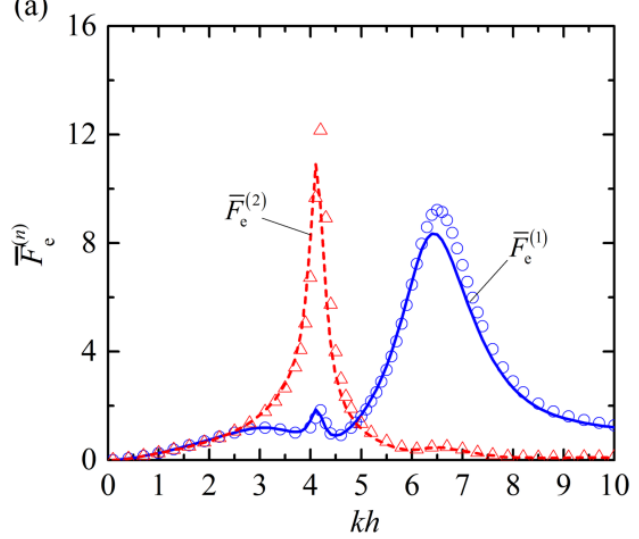

(c)

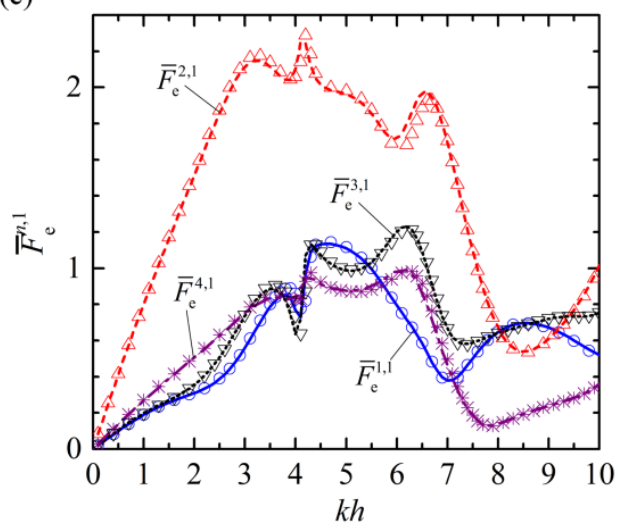

(e)

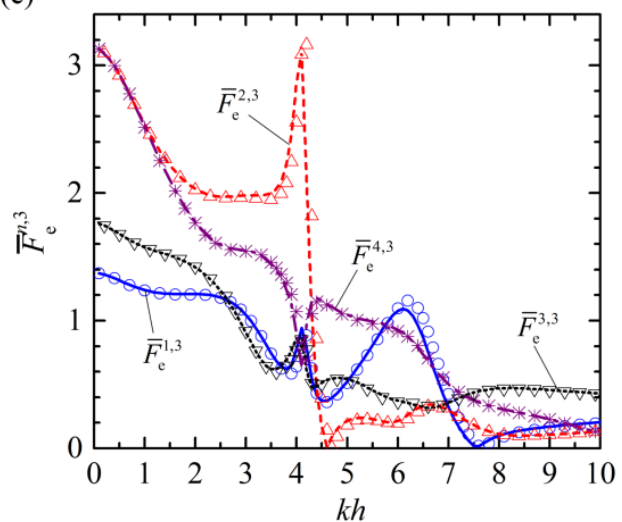

(g)

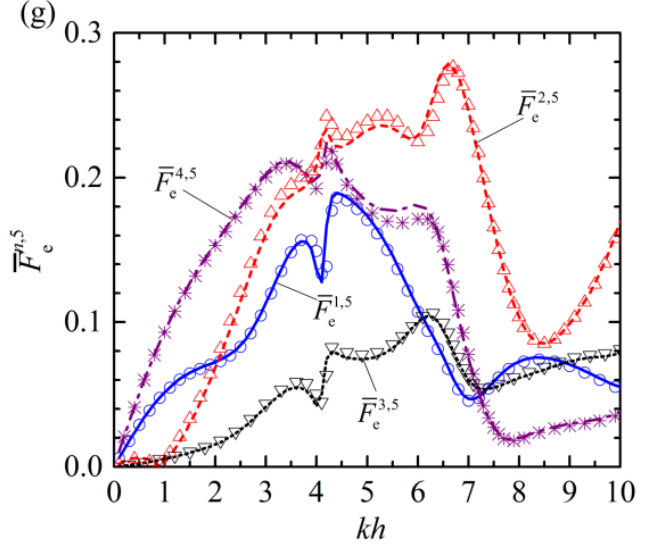

(b)

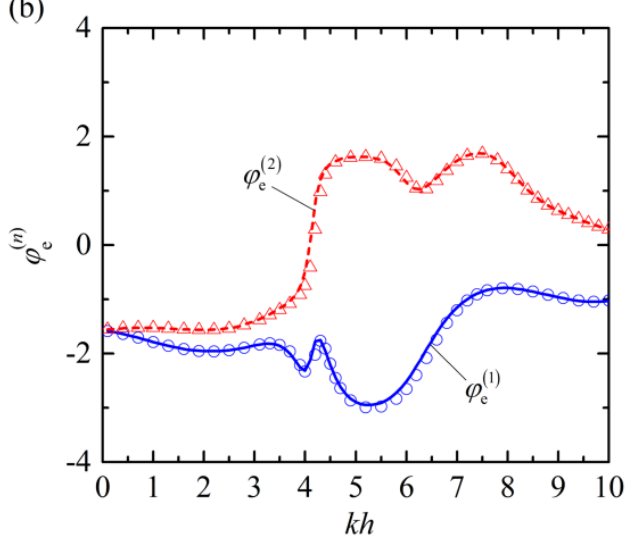

(d)

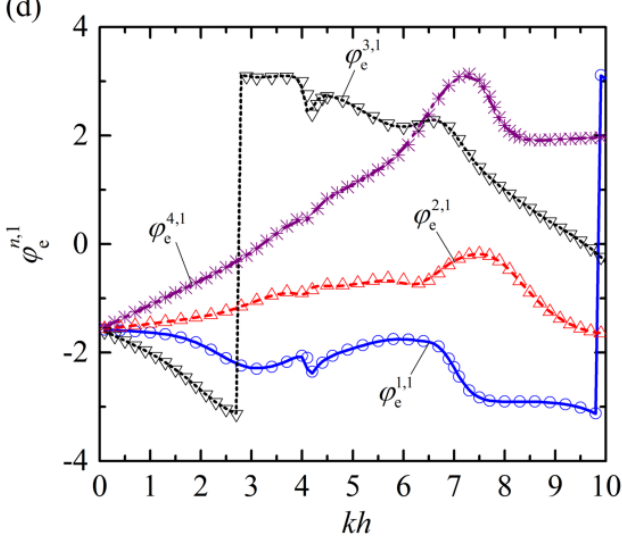

(f)

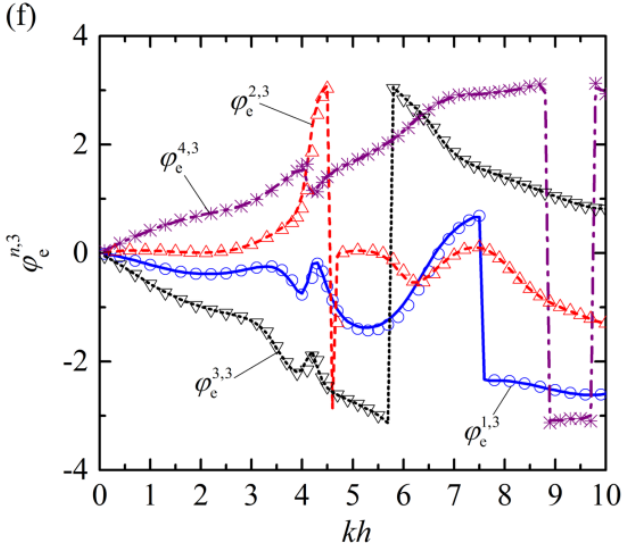

(h)

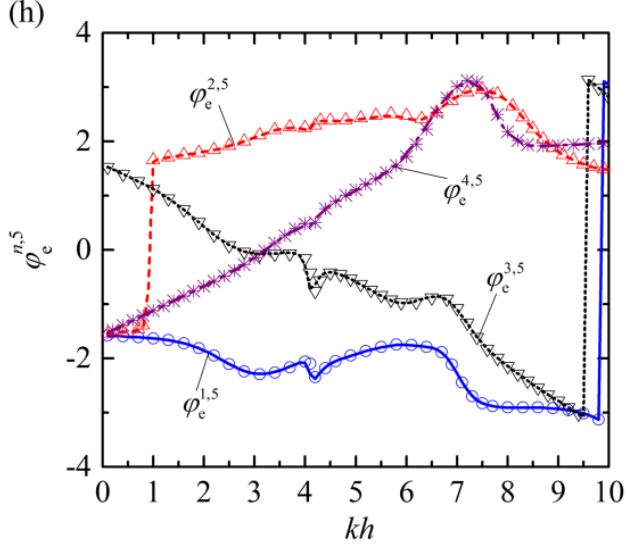

Fig. 5. Wave excitation volume fluxes and excitation forces acting on Floats 1 4 in surge, heave 
$1 \quad$ and pitch modes for $\beta=\pi / 6$. (a) $\bar{F}_{\mathrm{e}}^{(n)}$; (b) $\varphi_{\mathrm{e}}^{(n)}$; (c) $\bar{F}_{\mathrm{e}}^{n, 1}$; (d) $\varphi_{\mathrm{e}}^{n, 1}$; (e) $\bar{F}_{\mathrm{e}}^{n, 3}$; (f) $\varphi_{\mathrm{e}}^{n, 3} ;$ (g)

$$
\bar{F}_{\mathrm{e}}^{n, 5} ;(\mathrm{h}) \varphi_{\mathrm{e}}^{n, 5} \text {. lines: semi-analytical results; symbols: numerical results. }
$$

3

4

5

(a)

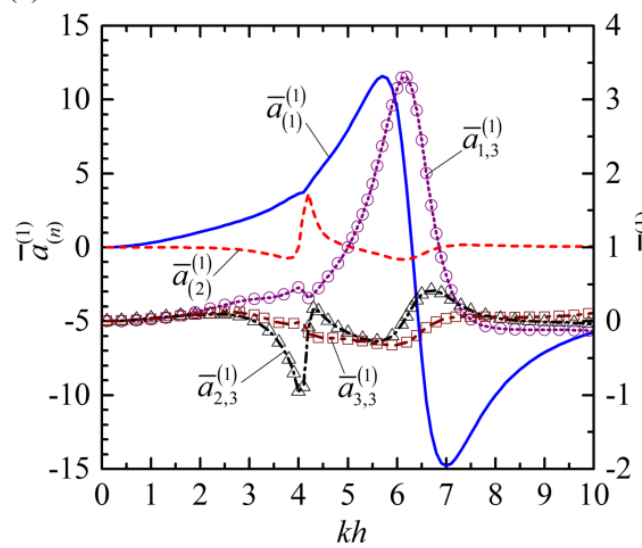

(c)

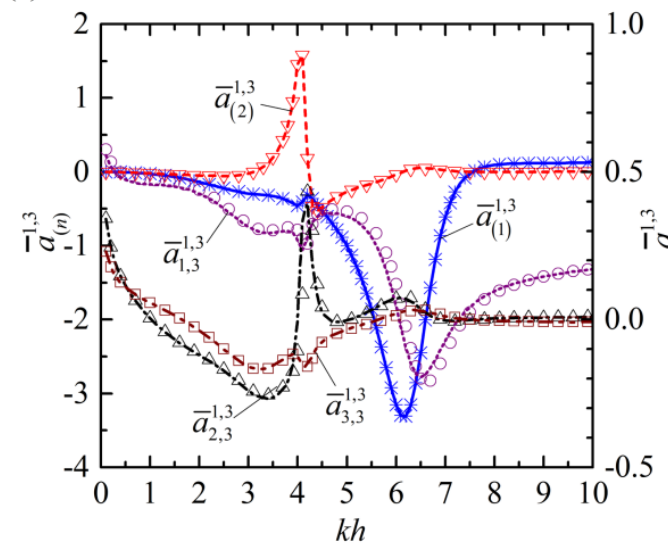

(e)

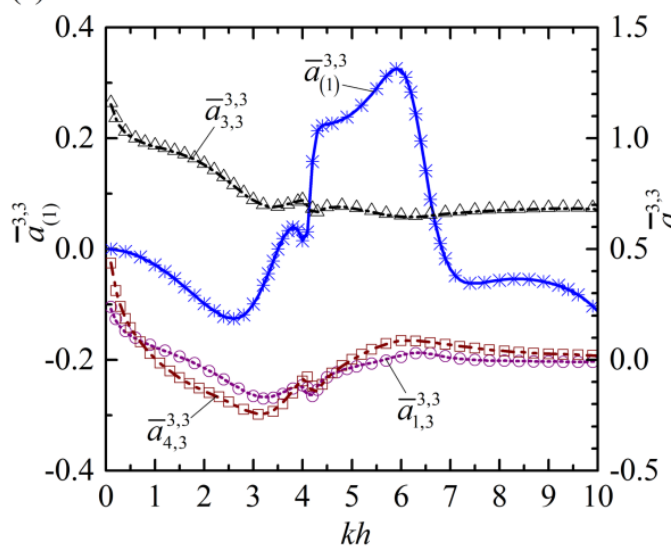
farm.

\subsection{Wave radiation}

(b)

(f)

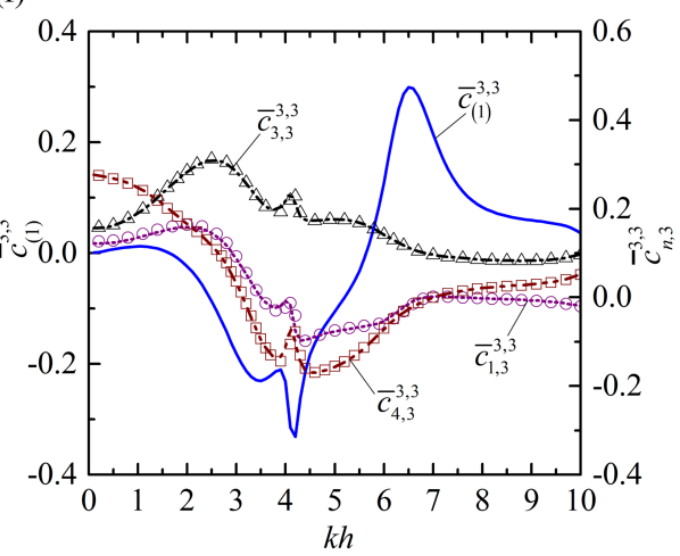

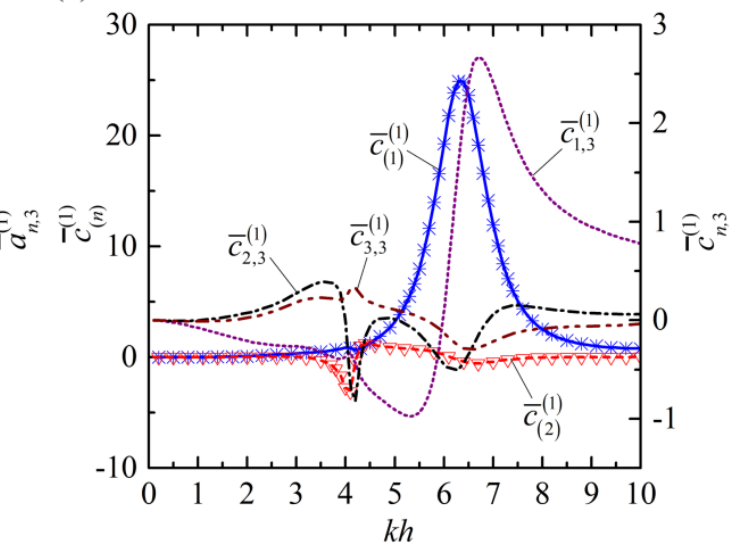

(d)

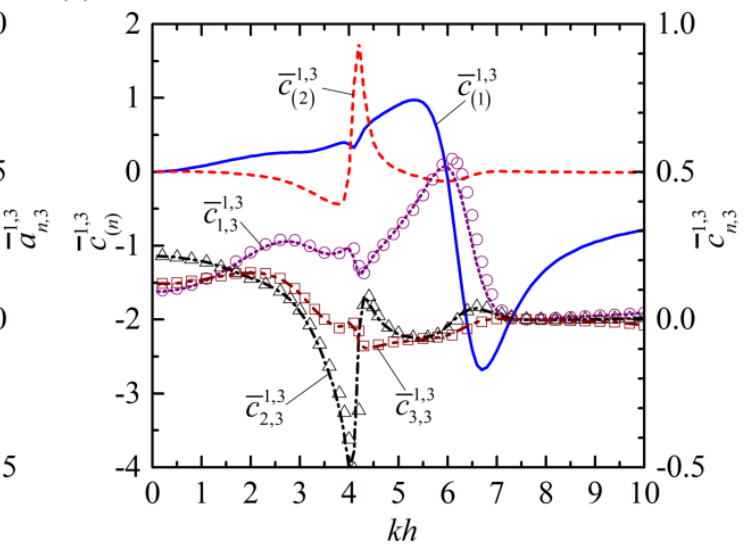

The good agreement between the semi-analytical results and numerical ones verifies the correctness of the semi-analytical model in solving wave diffraction problem of the hybrid wave

The results for wave radiation problem are illustrated in Fig. 6. 
1 Fig. 6. Hydrodynamic coefficients of Float 1 and Float 3. (a) $\bar{a}_{(n)}^{(1)}$ and $\bar{a}_{n, 3}^{(1)}$; (b) $\bar{c}_{(n)}^{(1)}$ and $\bar{c}_{n, 3}^{(1)}$;

(c) $\bar{a}_{(n)}^{1,3}$ and $\bar{a}_{n, 3}^{1,3}$;(d) $\bar{c}_{(n)}^{1,3}$ and $\bar{c}_{n, 3}^{1,3}$; (e) $\bar{a}_{(1)}^{3,3}$ and $\bar{a}_{n, 3}^{3,3}$;(f) $\bar{c}_{(1)}^{3,3}$ and $\bar{c}_{n, 3}^{3,3}$. lines: semi-analytical results using direct method; symbols: semi-analytical results using Haskind relation $\left(\bar{a}_{n, 3}^{(1)}, \bar{a}_{\left(n^{\prime}\right)}^{n, 3}, \bar{c}_{\left(n^{\prime}\right)}^{(n)}\right)$ and numerical results $\left(\bar{a}_{n, 3}^{n^{\prime}, 3}, \bar{c}_{n, 3}^{n^{\prime}, 3}\right)$. According to Eqs. (31) (33), there are relationships

$$
\begin{gathered}
a_{n, i}^{\left(n^{\prime}\right)}=-a_{\left(n^{\prime}\right)}^{n, i}, \quad c_{n, i}^{\left(n^{\prime}\right)}=-c_{\left(n^{\prime}\right)}^{n, i}, \\
a_{n, i}^{n^{\prime}, i^{\prime}}=a_{n^{\prime}, i^{\prime}}^{n, i}, \quad c_{n, i}^{n^{\prime}, i^{\prime}}=c_{n^{\prime}, i^{\prime}}^{n, i},
\end{gathered}
$$

which are also satisfied as shown in Fig. 6.

The good agreement between the semi-analytical results using different approaches and numerical ones shows that the semi-analytical model works pretty well in dealing with wave radiation problem by the hybrid wave farm.

\section{Results and discussion}

The geometries of OWCs and PAs used in the hybrid wave farms to be studied are selected as those previously adopted by Nader (2013), Konispoliatis and Mavrakos (2016) and Göteman (2017), respectively. Details of the parameters are given in Table 3. The reference length is chosen as $R_{0}=0.2 h$.

Table 3. Basic parameters of the OWCs and PAs.

\begin{tabular}{cccc} 
Float & $R_{n} / h$ & $R_{\mathrm{i}, n} / h$ & $d_{n} / h$ \\
\hline PAs & 0.14 & - & 0.024 \\
OWCs & 0.25 & 0.2 & 0.2 \\
\hline
\end{tabular}

Two different array configurations of a hybrid wave farm consisting of two OWCs and two PAs, denoted as \#H1 and \#H2, respectively (Fig. 7), will be examined in this section. The OWCs and PAs are placed at the angles of a square. The distance between the devices in the same row or column is denoted by $L$. 
(a)
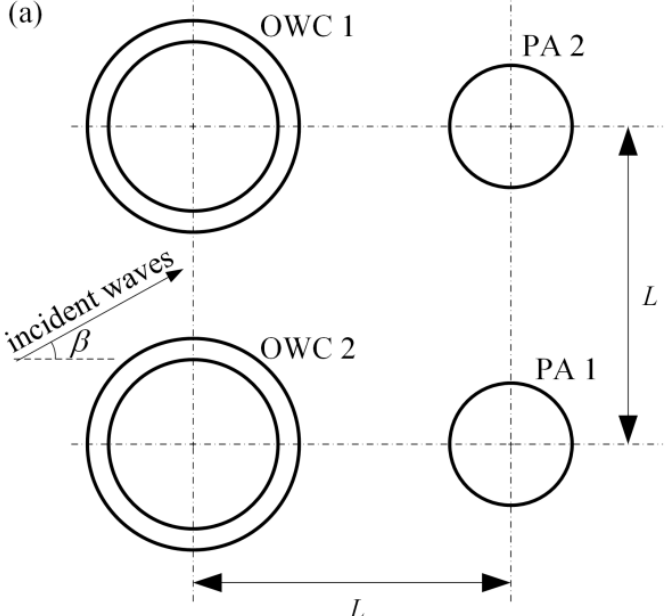

(b)

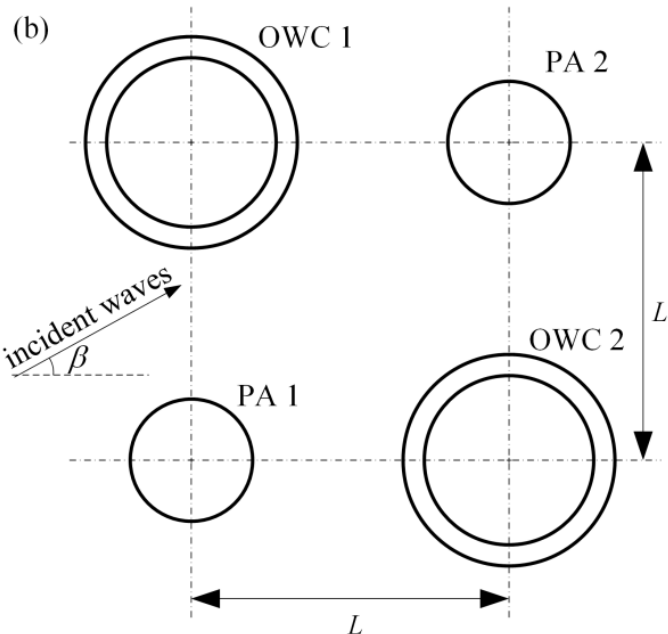

Fig. 7. Two configurations of the hybrid wave farm consisting of two OWCs and two PAs: (a)\# $\mathrm{H} 1$; (b) \# H2.

\subsection{Effect of incident wave direction}

In this subsection, the effect of the incident wave direction $(\beta)$ on the hydrodynamic performance of the hybrid wave farm is studied. The hydrodynamic coefficients are independent of $\beta$, as indicated in Sections 2 4; nevertheless, the wave excitation volume flux/forces are strongly dependent upon $\beta$.

Figure 8 presents the response of the wave excitation volume flux and heave wave excitation forces exerted on the OWCs/PAs in the hybrid wave farm $\# \mathrm{H} 1$ for different $\beta$ and $k h, L / h=0.75$. Since \#H1 is symmetric with respect to the $x$-axis, wave conditions with $\beta$ ranging from 0 to $\pi$ can be used to represent the wave incoming from all directions. It can be learnt from Figs. $8 \mathrm{a}$ and $8 \mathrm{~b}$ that $\bar{F}_{\mathrm{e}}^{(1)}$ and $\bar{F}_{\mathrm{e}}^{(2)}$ are both independent of $\beta$ for $k h<2.5$. For $k h<1.5$ and $k h>5.5$, both $\bar{F}_{\mathrm{e}}^{(1)}$ and $\bar{F}_{\mathrm{e}}^{(2)}$ are less than 1.0 regardless of $\beta$. Effect of $\beta$ mainly occurs at $2.5<k h<5.0$. When incident waves come from either the side of the two OWCs or the side of the two PAs, i.e., $\beta=0$ and $\pi$, the frequency response of $\bar{F}_{\mathrm{e}}^{(1)}$, i.e., $\bar{F}_{\mathrm{e}}^{(1)}-k h$ curve, reaches the peak value of 9.13 and 10.68 , respectively, both at $k h=3.4$. For $0.25 \pi<\beta<0.75 \pi$, the main peak value of $\bar{F}_{\mathrm{e}}^{(1)}$ occurs around $k h$ $=3.1$. This may be explained from the view of the "effective length" of wave diffraction distance. The incident and diffracted waves (including from the other WECs) act on the WEC simultaneously. For $\beta=0$ and $\pi$, such diffracted waves from PA 2 contribute a great deal to $\bar{F}_{\mathrm{e}}^{(1)}$; thus, the effective diffracted length is recognized as the distance between OWC 1 and PA 2, i.e., $L$. While for $0.25 \pi<\beta<0.75 \pi$, the waves diffracted from PA 1 play more important roles in $\bar{F}_{\mathrm{e}}^{(1)}$, leading to a larger effective diffracted length, i.e., $\sqrt{2} L$. The larger the wave diffraction distance, the incident and diffracted waves are more likely to stack up and boost up at a smaller $k h$. This 
1 rule also applies to $\bar{F}_{\mathrm{e}}^{(2)}$, as given in Fig. 8b. Different from the strong dependence of the peak

2 value of $\bar{F}_{\mathrm{e}}^{(1)}-k h$ curve on $\beta$, the peak values of the frequency response of $\bar{F}_{\mathrm{e}}^{(2)}$ for

$30.25 \pi<\beta<0.75 \pi$ are all around 13.3. Because of the symmetrical property of \#H1, the heave wave 4 excitation forces/volume flux of OWC 1 and PA 1 are the same as those of OWC 2 and PA 2 , 5 respectively, when $\beta=0$ and $\pi$, which is readily seen in Fig. 8.

6
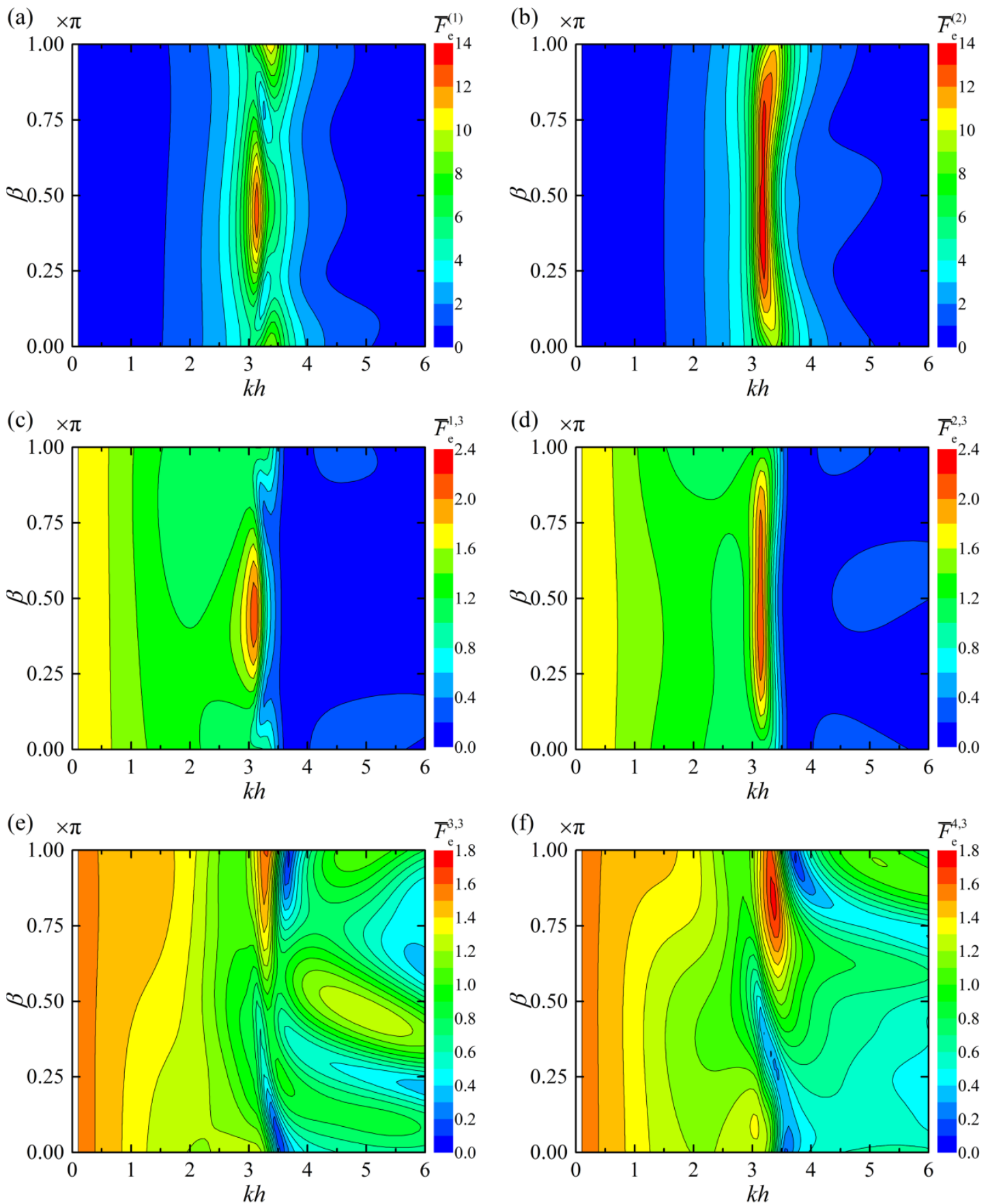

Fig. 8. Wave excitation volume flux/forces of the WECs in the hybrid wave farm \#H1 versus $k h$ and $\beta$ for $L / h=0.75$ : (a) $\bar{F}_{\mathrm{e}}^{(1)}$; (b) $\bar{F}_{\mathrm{e}}^{(2)}$; (c) $\bar{F}_{\mathrm{e}}^{1,3}$; (d) $\bar{F}_{\mathrm{e}}^{2,3}$; (e) $\bar{F}_{\mathrm{e}}^{3,3}$; (f) $\bar{F}_{\mathrm{e}}^{4,3}$. 
1

2

Figures $8 \mathrm{c}$ and $8 \mathrm{~d}$ illustrate that for most $\beta$, as $k h$ increases from 0.1 to 6.0 , both of the excitation heave forces acting on the two OWCs $\left(\bar{F}_{\mathrm{e}}^{1,3}\right.$ and $\left.\bar{F}_{\mathrm{e}}^{2,3}\right)$ first decrease from 1.76 and then increase to a peak, after which they rapidly drop to zero at $k h=3.7$. As $k h$ keeps increasing, $\bar{F}_{\mathrm{e}}^{1,3}$ and $\bar{F}_{\mathrm{e}}^{2,3}$ rise and stay at a relatively stable low value at $4.0<k h<6.0$. The peak values of $\bar{F}_{\mathrm{e}}^{1,3}-k h$ curves are $1.03,1.68,2.10,1.34$ and 1.21 at $k h=3.14,3.08,3.08,3.02$, and 3.20 , respectively, for $\beta=0,0.25 \pi, 0.5 \pi, 0.75 \pi$ and $\pi$. While the peaks of $\bar{F}_{\mathrm{e}}^{2,3}-k h$ curves for $0.25 \pi<\beta<0.75 \pi$ almost coincide with each other at $k h=3.14$, with peak values around 2.10.

As shown in Figs. 8e and 8f, the heave excitation forces acting on the two PAs are strongly affected by $\beta$. Rapid drops and rises of $\bar{F}_{\mathrm{e}}^{3,3}$ and $\bar{F}_{\mathrm{e}}^{4,3}$ versus $k h$ occur at $2.5<k h<4.0$, and the changing amplitude and $k h$ for which such rapid changes occur are both strongly influenced by $\beta$. For long waves, i.e., $1.0<k h<2.0$, generally the larger the value of $\beta$, the larger the $\bar{F}_{\mathrm{e}}^{3,3}$. As a comparison, although the largest value of $\bar{F}_{\mathrm{e}}^{4,3}$ (see Fig. 8f) in long waves also appears at $\beta=\pi$, the smallest occurs at $0.25 \pi<\beta<0.5 \pi$ rather than $\beta=0$, which is induced by the shading effect of OWC 1 and OWC 2 in front of PA 2. For short waves, i.e., $4.2<k h<5.9, \bar{F}_{\mathrm{e}}^{4,3}$ around $\beta=0.96 \pi$ is much larger than those in other situations. Whereas $\bar{F}_{\mathrm{e}}^{3,3}$ at $\beta \approx 0.5 \pi$ is even larger than that for $\beta=0.96 \pi$, which could be due to the large waves diffracted from OWC 2 .

The wave excitation volume flux and heave wave excitation forces acting on the OWCs/PAs in hybrid wave farm $\# \mathrm{H} 2$ for different incident wave directions and wave numbers are plotted in Fig. 9. Because of the symmetrical deployment of $\# \mathrm{H} 2,-0.25 \pi \leq \beta \leq 0.25 \pi$ is selected to represent the entire range of incident wave directions (from $-\pi$ to $\pi$ ). Due to the symmetry, we have $\bar{F}_{\mathrm{e}}^{(1)}=$ $\bar{F}_{\mathrm{e}}^{(2)}$, and $\bar{F}_{\mathrm{e}}^{1,3}=\bar{F}_{\mathrm{e}}^{2,3}$ for $\beta=\pi / 4$, which is readily seen in Figs. 9a $\sim 9 \mathrm{~d}$. As $\beta$ increases from $-0.25 \pi$ to $0.25 \pi$, the peak value of the frequency response of $\bar{F}_{\mathrm{e}}^{(1)}$ and the corresponding $k h$ both turn larger and larger. The peak value of $\bar{F}_{\mathrm{e}}^{(1)}$ can be larger than 16.0, which occurs at $k h=3.32$ when $0.18 \pi \leq \beta \leq 0.25 \pi$. 

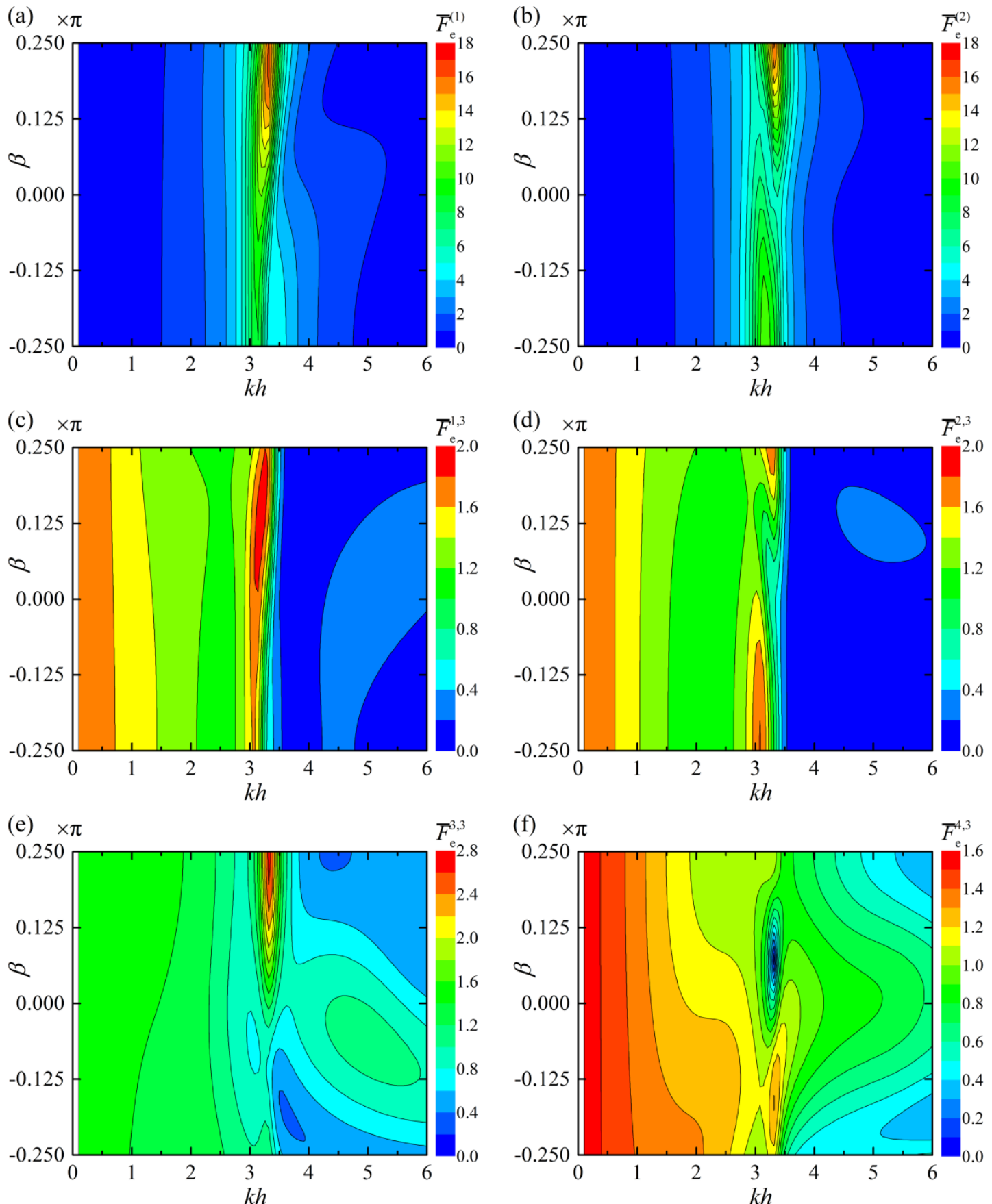

Fig. 9. Wave excitation volume flux/forces of the WECs in the hybrid wave farm \#H2 versus $k h$ and $\beta$ for $L / h=0.75$ : (a) $\bar{F}_{\mathrm{e}}^{(1)}$; (b) $\bar{F}_{\mathrm{e}}^{(2)}$; (c) $\bar{F}_{\mathrm{e}}^{1,3}$; (d) $\bar{F}_{\mathrm{e}}^{2,3}$; (e) $\bar{F}_{\mathrm{e}}^{3,3}$; (f) $\bar{F}_{\mathrm{e}}^{4,3}$.

The behavior of $\bar{F}_{\mathrm{e}}^{(2)}$ as given in Fig. $9 \mathrm{~b}$ is rather different from that of $\bar{F}_{\mathrm{e}}^{(1)}$. There are two peaks in the contour-plot of $\bar{F}_{\mathrm{e}}^{(2)}$ in the plane of $k h-\beta$ : one appears at $k h=3.14, \beta=-0.25 \pi$; the other one occurs at $k h=3.32, \beta=0.25 \pi$. Note that for $\beta=-0.25 \pi$, the incident waves propagate along 8 the diagonal line from OWC 1 to OWC 2, nevertheless it is found that $\bar{F}_{\mathrm{e}}^{(2)}>\bar{F}_{\mathrm{e}}^{(1)}$ for $2.7<k h<3.5$. 
1 That is to say, the excitation volume flux of lee-side OWC is larger that of the sea-side one in

2 these wave conditions. This could be caused by the diffracted waves from the two PAs toward

3 OWC 2. A similar behavior is also observed with $\bar{F}_{\mathrm{e}}^{1,3}$ and $\bar{F}_{\mathrm{e}}^{2,3}$ (Figs. 9c and 9d).

Because of the symmetry of $\# \mathrm{H} 2, \quad \bar{F}_{\mathrm{e}}^{3,3}=\bar{F}_{\mathrm{e}}^{4,3}$ is satisfied for $\beta=-0.25 \pi$ (Figs. 9e and 9f). For

$5 k h<2.0$, as $\beta$ increases from $-0.25 \pi$ to $0.25 \pi, \bar{F}_{\mathrm{e}}^{3,3}$ increases, whereas $\bar{F}_{\mathrm{e}}^{4,3}$ decreases. For $2.5<$

$k h<4.0$, the peak value of $\bar{F}_{\mathrm{e}}^{4,3}$ is a mere 1.30 at $k h=3.32, \beta=-0.16 \pi$, in contrast to the peak value

7 of $\bar{F}_{\mathrm{e}}^{3,3}$, which is 2.69 at $k h=3.32, \beta=0.25 \pi$. For $k h>4.0$, a peak of $\bar{F}_{\mathrm{e}}^{3,3}$ appears at $k h=4.99$,

$8 \beta=-0.06 \pi$. In the same range of $k h, \bar{F}_{\mathrm{e}}^{4,3}$ around $\beta=0$ is more likely to have a large value because

9 of the influence by the waves diffracted from OWC 2, and the smaller the $k h$, the larger the $\bar{F}_{\mathrm{e}}^{4,3}$.

To have a more clear view of the effect of $\beta$ on the wave excitation forces/volume flux, the results versus $\beta$ for two wave conditions, i.e., $k h=3.3$ and 5.0, for configurations $\# \mathrm{H} 1$ and $\# \mathrm{H} 2$ are also plotted in Figs. 10 and 11, respectively.

(a)

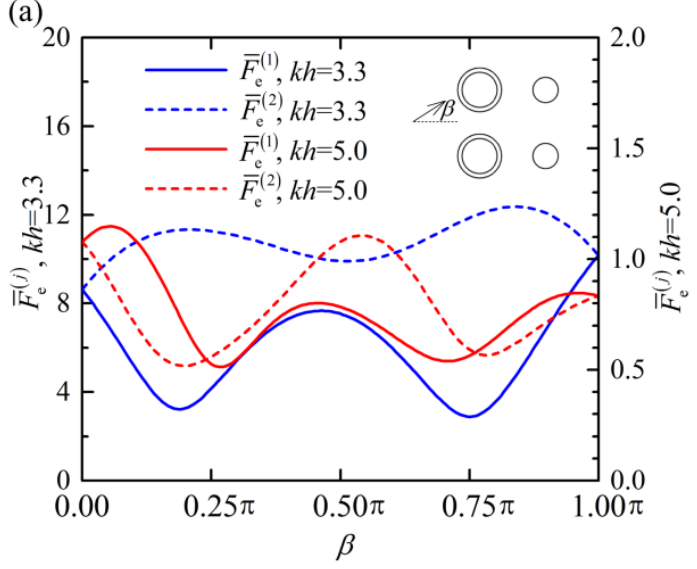

(b)

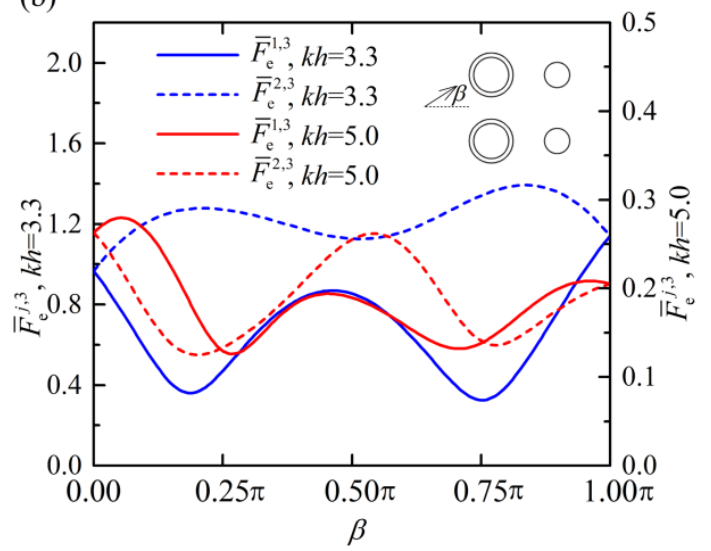

(c)

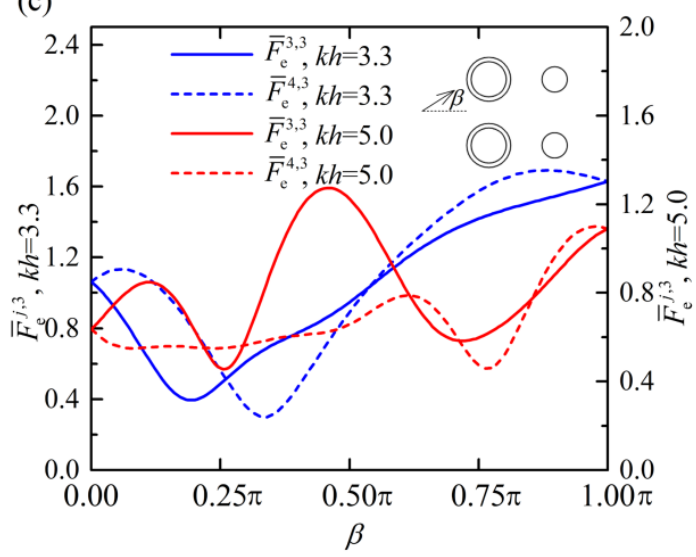

Fig. 10. Wave excitation volume flux/forces of the WECs in the hybrid wave farm \#H1 versus $\beta$ for $k h=3.3$ and $5.0, L / h=0.75$

(a) $\bar{F}_{\mathrm{e}}^{(1)}, \bar{F}_{\mathrm{e}}^{(2)}$;

(b) $\bar{F}_{\mathrm{e}}^{1,3}, \bar{F}_{\mathrm{e}}^{2,3}$;

(c) $\bar{F}_{\mathrm{e}}^{3,3}, \bar{F}_{\mathrm{e}}^{4,3}$. 
(a)

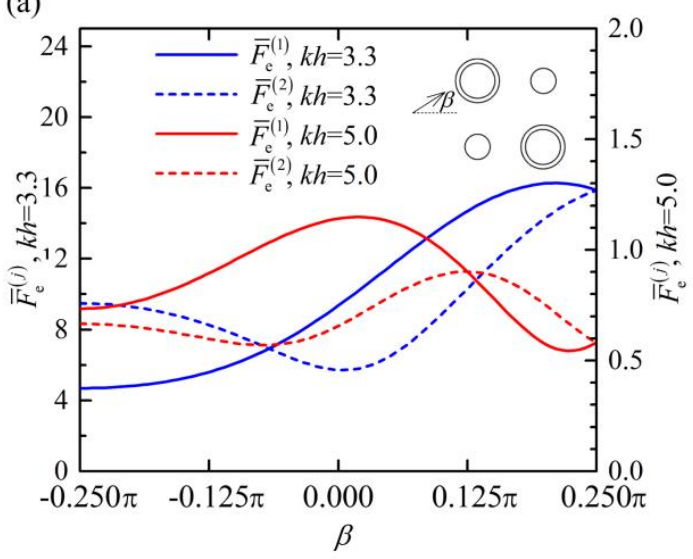

(b)

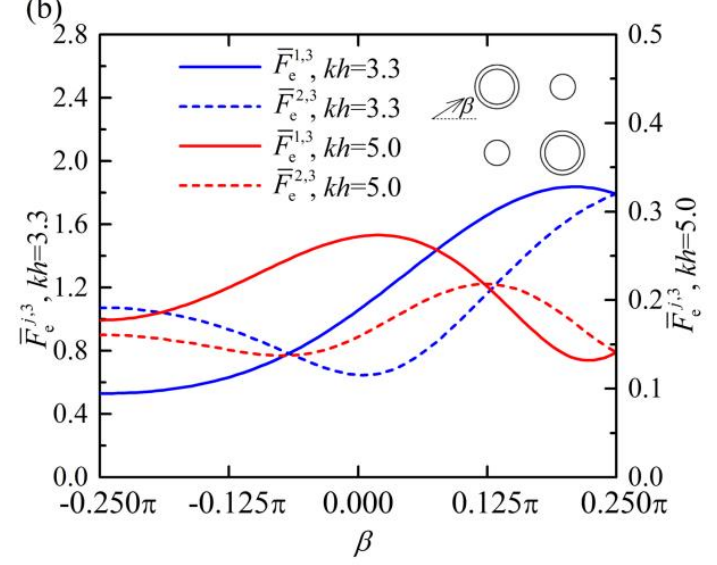

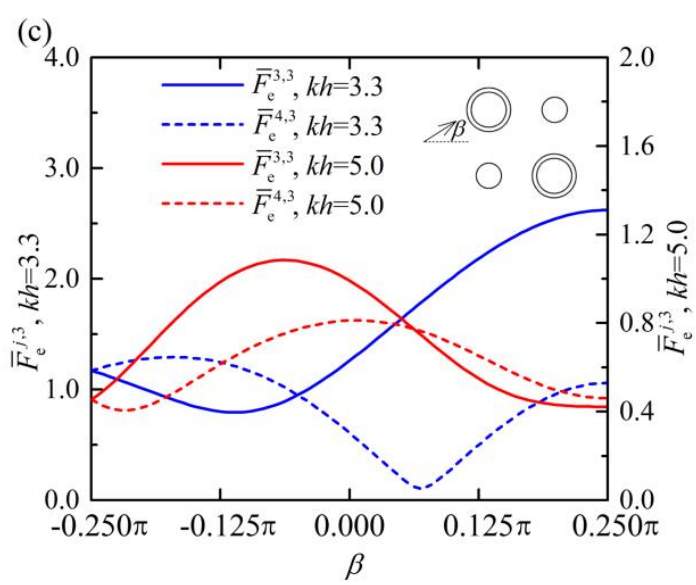

For \#H1 in wave conditions with $k h=3.3$ (see Fig.10a), the wave excitation volume flux of sea-side OWC (OWC 2) is always larger than that of the lee-side one (OWC 1), i.e., $\bar{F}_{\mathrm{e}}^{(2)}>\bar{F}_{\mathrm{e}}^{(1)}$. The largest value of $\bar{F}_{\mathrm{e}}^{(2)}$ is 12.36 occurring at $\beta=0.84 \pi$, and the smallest value of $\bar{F}_{\mathrm{e}}^{(1)}$ is only 2.88 when $\beta=0.75 \pi$. Note the largest value of $\bar{F}_{\mathrm{e}}^{(1)}$ is 10.17 , occurring at $\beta=\pi$, where due to the symmetrical property of \#H1 about the $x$-axis, $\bar{F}_{\mathrm{e}}^{(2)}$ has the same value as well. Variations of $\bar{F}_{\mathrm{e}}^{(1)}$ and $\bar{F}_{\mathrm{e}}^{(2)}$ with $\beta$ change a lot for different wave numbers. For $k h=5.0$, the wave excitation volume flux of lee-side OWC is found to be larger than that of the sea-side one when $0<\beta<0.24 \pi$ and $0.76 \pi<\beta<1.0 \pi$. The largest and the least values of the wave excitation volume flux in \#H1 both happen on the lee-side OWC, which are $\bar{F}_{\mathrm{e}}^{(1)}=1.15$ and 0.51 , occurring at $\beta=0.05 \pi$ and $0.27 \pi$, respectively.

Variation of $\bar{F}_{\mathrm{e}}^{j, 3}(j=1,2)$ with $\beta$ as shown in Fig.10b presents a similar trend with that of 
$\bar{F}_{\mathrm{e}}^{(j)}-\beta$. Nevertheless, variation of $\bar{F}_{\mathrm{e}}^{j, 3}(j=3,4)$ with $\beta$ as given in Fig. $10 \mathrm{c}$ is rather different compared with $\bar{F}_{\mathrm{e}}^{(j)}-\beta$ and $\bar{F}_{\mathrm{e}}^{j, 3}(j=1,2)-\beta$. For $k h=3.3, \bar{F}_{\mathrm{e}}^{3,3}$ and $\bar{F}_{\mathrm{e}}^{4,3}$ reach their least values at $\beta=0.19 \pi$ and $0.33 \pi$, respectively. As $\beta$ increases from $0.33 \pi$ to $\pi$, the heave excitation forces acting on the two PAs both increase and achieve their highest level at $0.85 \pi<\beta \leq \pi$. For $k h=5.0$ with $\beta$ varying from 0 to $0.5 \pi, \bar{F}_{\mathrm{e}}^{4,3}$ stays at a rather stable level around 0.6 , whereas $\bar{F}_{\mathrm{e}}^{3,3}$ dramatically fluctuates between 0.46 and 1.27. This can be explained by the strong shadowing effect of the two OWCs in front of the two PAs.

As given in Fig.11, for \#H2 in which the two OWCs and PAs are deployed diagonally, as the incident direction of the waves with $k h=3.3$ increases from $-0.25 \pi$ to $0.25 \pi$, the excitation volume flux of sea-side OWC, i.e., $\bar{F}_{\mathrm{e}}^{(1)}$, increases monotonically from 4.69 and reaches its largest value of 16.27 at $0.2 \pi<\beta \leq 0.25 \pi$. The peak value of $\bar{F}_{\mathrm{e}}^{(2)}$ occurs in such range of $\beta$ as well $(\beta=0.25 \pi$ exactly), while the least one is obtained at $\beta=0.01 \pi$, rather than $\beta=-0.25 \pi$. For $k h=5.0$, both $\bar{F}_{\mathrm{e}}^{(1)}$ and $\bar{F}_{\mathrm{e}}^{(2)}$ reach their minimum at $\beta=0.25 \pi$. The results of $\bar{F}_{\mathrm{e}}^{j, 3}(j=1,2)$ varying with $\beta$ for $k h=3.3$ and 5.0 as plotted in Fig. 11b show similar trends with those of $\bar{F}_{\mathrm{e}}^{(j)}-\beta$. As illustrated in Fig.11c, for $k h=3.3$, the heave excitation force acting on sea-side PA (PA 1), i.e., $\bar{F}_{\mathrm{e}}^{3,3}$, reaches the maximum value 2.62 at $\beta=0.25 \pi$ due to strong wave reflection from the two OWCs. The maximum value of $\bar{F}_{\mathrm{e}}^{4,3}$ for $k h=3.3$ is observed at $\beta=-0.17 \pi$. Note the minimum value of $\bar{F}_{\mathrm{e}}^{4,3}$ occurring at $\beta=0.07 \pi$ for $k h=3.3$ is merely 0.11 . For such situation, incident waves are nearly entirely reflected by the two OWCs and the sea-side PA, and only a tiny fraction propagate through lee-side PA. The variation trend of $\bar{F}_{\mathrm{e}}^{3,3}-\beta$ for $k h=5.0$ is found to be approximately opposite of that for $k h=3.3$, revealing that the diffraction effect of the WECs for different $\beta$ is significantly dependent on wave numbers.

From the over view of Fig.10 for $k h=3.3, \bar{F}_{\mathrm{e}}^{(j)}$ and $\bar{F}_{\mathrm{e}}^{j, 3}$ are all at a pretty large level at $\beta=\pi$, where more wave power could be absorbed by hybrid wave farm \#H1. For \#H2 with $k h=3.3$, $\beta=0.25 \pi$ might be a better choice, for $\bar{F}_{\mathrm{e}}^{(j)}$ and $\bar{F}_{\mathrm{e}}^{j, 3}$ are rather large with such wave incident direction as shown in Fig. 11.

\subsection{Effect of WEC spacing}

It will be shown in this section that the device spacing, i.e., the distance between two WECs in the same row or column $(L)$, also plays a significant role. We have learnt from Section 6.1 that $\beta=\pi$ and $0.25 \pi$ are good choices for hybrid wave farms $\# \mathrm{H} 1$ and $\# \mathrm{H} 2$, respectively, for certain 
1 specified wave conditions (e.g., $k h=3.3$ ) and $L / h=0.75$. In this section we discuss the effect of $L / h$

2 on the performance of wave farms with $\beta=\pi$ and $0.25 \pi$ employed for $\# \mathrm{H} 1$ and \#H2, separately.

3

4

5

6

7

8
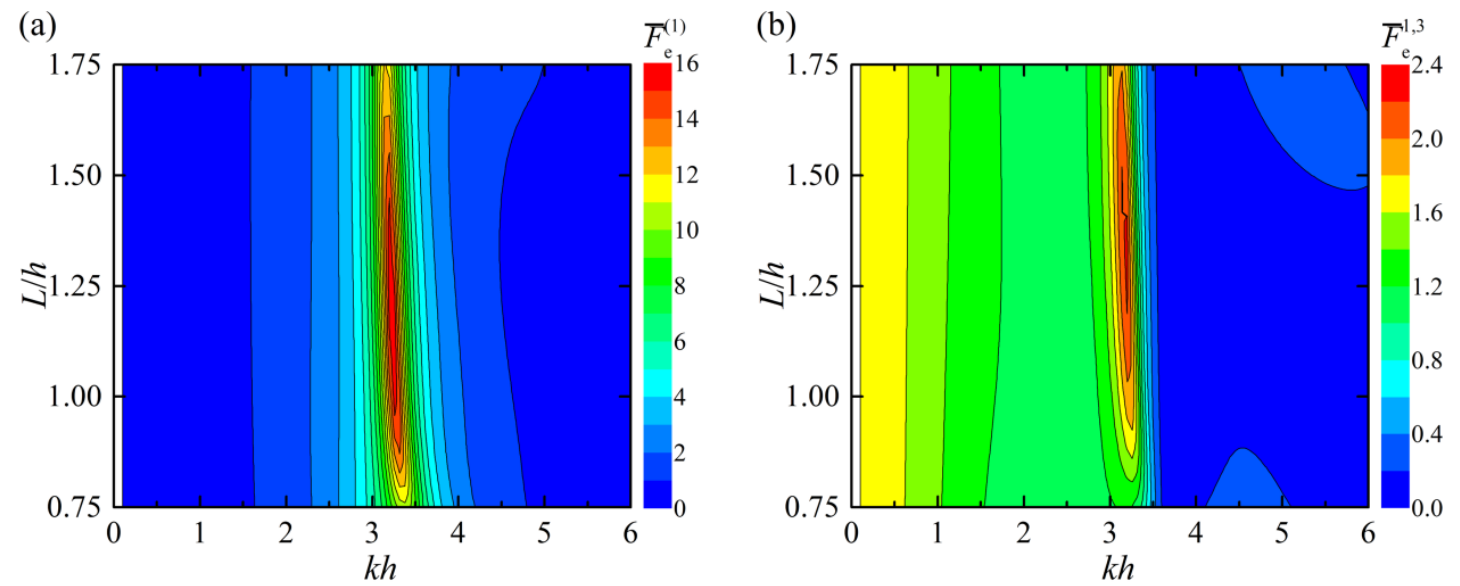

(c)

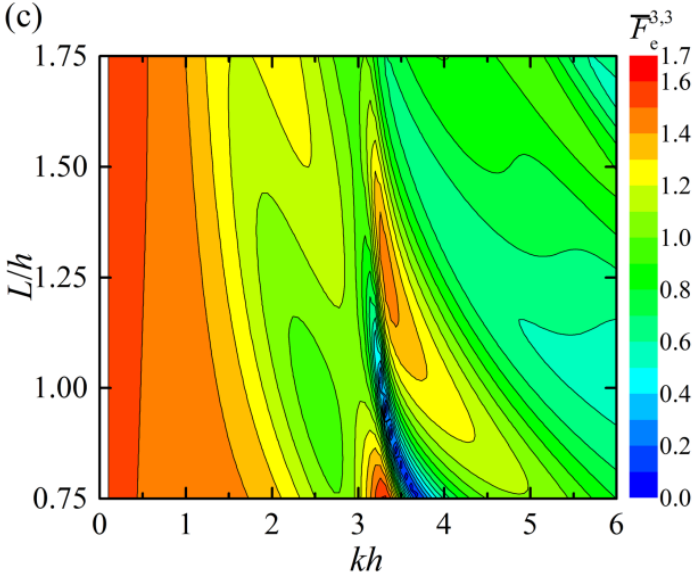

Fig. 12. Wave excitation volume flux/forces of the WECs in the hybrid wave farm \#H1 versus

$$
k h \text { and } L / h \text { for } \beta=\pi \text { : (a) } \bar{F}_{\mathrm{e}}^{(1)} \text {; (b) } \bar{F}_{\mathrm{e}}^{1,3} \text {; (c) } \bar{F}_{\mathrm{e}}^{3,3} \text {. }
$$



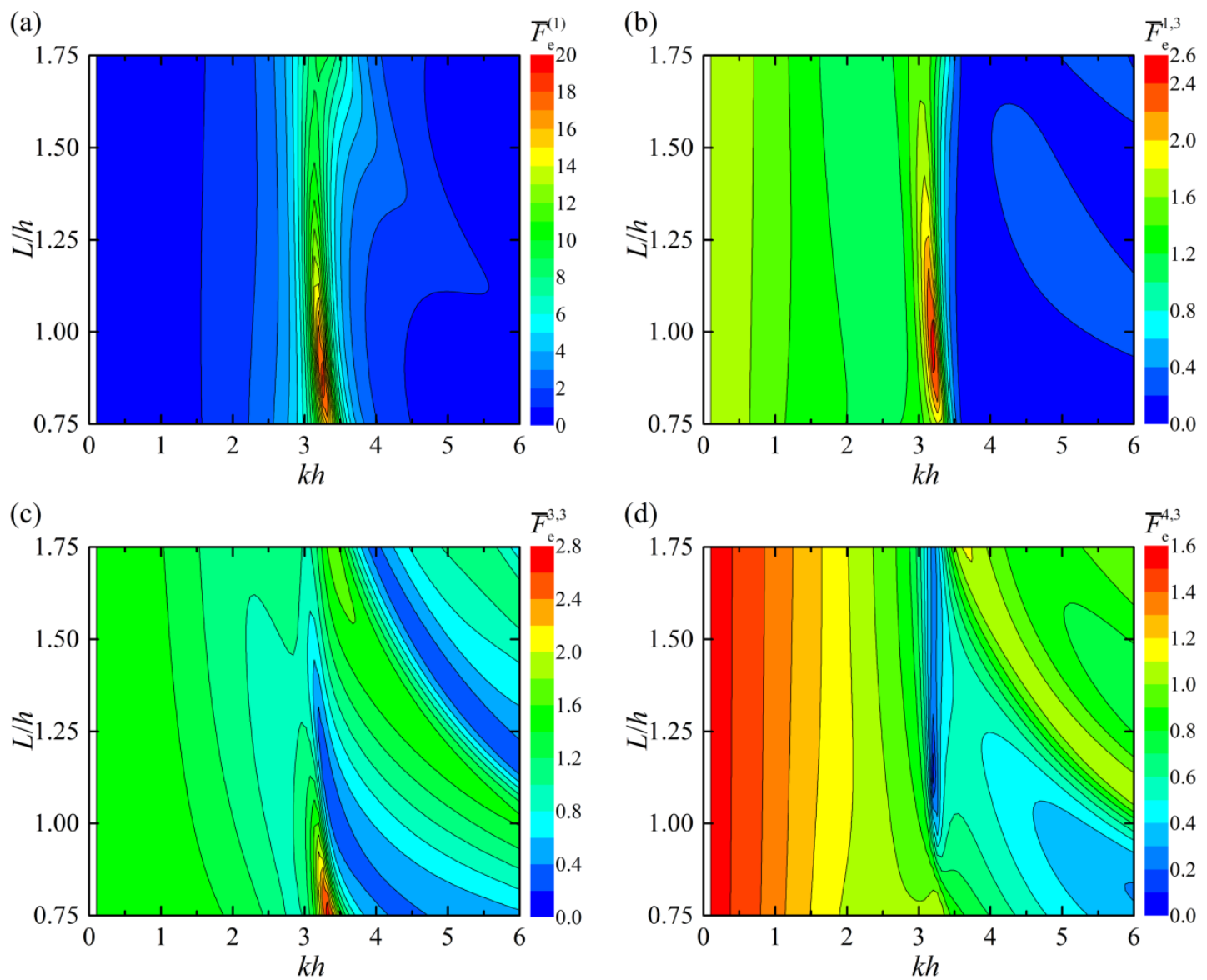

Fig. 13. Wave excitation volume flux/forces of the WECs in the hybrid wave farm \#H2 versus

$$
k h \text { and } L / h \text { for } \beta=0.25 \pi \text { : (a) } \bar{F}_{\mathrm{e}}^{(1)} \text {; (b) } \bar{F}_{\mathrm{e}}^{1,3} \text {; (c) } \bar{F}_{\mathrm{e}}^{3,3} \text {;(d) } \bar{F}_{\mathrm{e}}^{4,3} \text {. }
$$

As illustrated in Fig.12, $\bar{F}_{\mathrm{e}}^{(1)}$ is strongly influenced by wave number, especially when $k h$ ranges from 2.5 to 4.0 . For any specified value of $L / h$, there is a peak of frequency response of $\bar{F}_{\mathrm{e}}^{(1)}$ occurring at $3.0<k h<3.5$. Effect of $L / h$ is mainly observed on the peak of frequency response of $\bar{F}_{\mathrm{e}}^{(1)}$ in terms of the peak value and also the corresponding $k h$ where the peak occurs. The peak values of $\bar{F}_{\mathrm{e}}^{(1)}-k h$ curves for $L / h=0.75,1.0,1.25,1.5$ and 1.75 are $10.68,15.49,15.52,14.55$ and 12.13 , occurring at $k h=3.38,3.26,3.20,3.20$ and 3.14 , respectively. It is indicated that with increase of $L / h$, the peak value of $\bar{F}_{\mathrm{e}}^{(1)}-k h$ curve first increases and then decreases, whereas the $k h$ corresponding to the peak decreases all the time, reflecting the rule of wave diffraction distance as discussed previously in Section 6.1. A similar variation trend of the peak in terms of both peak amplitude and the corresponding $k h$ with $L / h$ also applies to $\bar{F}_{\mathrm{e}}^{1,3}$ (see Fig. 12b). It is also observed from the data of Fig. $12 \mathrm{~b}$ that $\bar{F}_{\mathrm{e}}^{1,3}$ vanishes at $k h=3.7$, regardless the value of $L / h$. For a 
1 given value of $k h$ between 0.1 and $6.0, \bar{F}_{\mathrm{e}}^{3,3}$ varies significantly with $L / h$ (see Fig. 12c),

2 indicating a strong dependence of $\bar{F}_{\mathrm{e}}^{3,3}$ on $L / h$. Take $k h=3.3$ as an example, as $L / h$ increases from

30.75 to $1.75, \bar{F}_{\mathrm{e}}^{3,3}$ decreases from 1.62 rapidly and then increases dramatically after reaching the

4 minimum value of 0.03 at $L / h=0.95$. As $L / h$ keeps increasing, a peak value of $\bar{F}_{\mathrm{e}}^{3,3}, 1.46$, is

5 obtained at $L / h=1.27$, after which $\bar{F}_{\mathrm{e}}^{3,3}$ turns smaller and smaller.

For $\# \mathrm{H} 2$ with $\beta=0.25 \pi$, incident waves propagate along the symmetrical line of the two OWCs; thus, $\bar{F}_{\mathrm{e}}^{(1)}$ and $\bar{F}_{\mathrm{e}}^{1,3}$ (Figs. 13a and 13b) also apply to OWC 2. Compared with the other values of $L / h, L / h=0.8$ and 0.87 lead to the larger peaks of the $\bar{F}_{\mathrm{e}}^{(1)}-k h$ and $\bar{F}_{\mathrm{e}}^{1,3}-k h$ curves, respectively, both occurring around $k h=3.3$. For such cases, the distance between OWC and PA (i.e., $L$ ) is approximately equal to a half of the wave length. Therefore, the diffracted waves from PAs could greatly contribute the improvement of both $\bar{F}_{\mathrm{e}}^{(1)}$ and $\bar{F}_{\mathrm{e}}^{1,3}$. Figures $13 \mathrm{c}$ and $13 \mathrm{~d}$ present the contour plots of the heave excitation forces acting on the sea-side and lee-side PAs, i.e., $\bar{F}_{\mathrm{e}}^{3,3}$ and $\bar{F}_{\mathrm{e}}^{4,3}$, respectively. For $2.0<k h<6.0$ and $0.75<L / h<1.75$, as shown in Fig. 13d, the largest value of $\bar{F}_{\mathrm{e}}^{4,3}$ is a mere 1.12. By contrast, in the same range of $k h$ and $L / h$, the peak value of $\bar{F}_{\mathrm{e}}^{3,3}$ reaches 2.69 at $k h=3.32$ and $L / h=0.76$.

Figures 14 and 15 show the hydrodynamic coefficients in heave mode for configurations \#H1 and \#H2, respectively. The variation of $\bar{a}_{(1)}^{(1)}$ with $k h$ presents a curve in "N" shape (Figs. 14a and 15a). For $k h$ tending to $0, \bar{a}_{(1)}^{(1)}$ tends to 0 too. As $k h$ increases, $\bar{a}_{(1)}^{(1)}$ first increases, up to a maximum at at $k h=3.0$, and then drops abruptly to negative values. As $k h$ keeps increasing, $\bar{a}_{(1)}^{(1)}$ increases again and tends to stay at a relatively stable level. As a comparison, the $\bar{c}_{(1)}^{(1)}-k h$ curve presents only one extreme (maximum), $\bar{c}_{(1)}^{(1)}$ is positive all the time, and $\bar{c}_{(1)}^{(1)}$ tends to 0 both for for $k h$ tending to 0 and $\infty$ (Figs. $14 \mathrm{~b}$ and $15 \mathrm{~b}$ ). It is observed that the peak of $\bar{c}_{(1)}^{(1)}$ and the jump of $\bar{a}_{(1)}^{(1)}$ occurs at the same wave condition $k h=3.2$. Furthermore, the peak of $\bar{c}_{(1)}^{(1)}$ bears relation to the jump behaviour of $\bar{a}_{(1)}^{(1)}$. Similar results have also been reported in radiation problems of oscillating bodies in channels (Linton and Evans, 1993) and open cylinders (Nokob and Yeung, 2015), in which heuristic arguments were given by plotting the trace of the complex force 
1 coefficient $\left(\bar{c}_{(1)}^{(1)}+\mathrm{i} \bar{a}_{(1)}^{(1)}\right.$ for the present case) in the radiation damping-added mass plane $\left(\bar{c}_{(1)}^{(1)}-\bar{a}_{(1)}^{(1)}\right.$

2 plane for the present case). Similar observations were reported by Sarkar et al. (2015) for the case

3 of a flap-type WEC in front of a vertical straight coast. The effect of $L / h$ on $\bar{a}_{(1)}^{(1)}$ and $\bar{c}_{(1)}^{(1)}$ is

4 mainly found at $3.0<k h<4.0$, where the sharp change of $\bar{a}_{(1)}^{(1)}$ and the peak of $\bar{c}_{(1)}^{(1)}$ occur. The

5 peak value of $\bar{c}_{(1)}^{(1)}$ for \#H2 with $L / h=1.0$ is 47.86 , much larger than that for \#H2 with any other

6 value of $L / h$ and also larger than that for \#H1. For the remaining wave conditions, $\bar{a}_{(1)}^{(1)}$ and $\bar{c}_{(1)}^{(1)}$

7 are found to be nearly independent of $L / h$. 
(a)

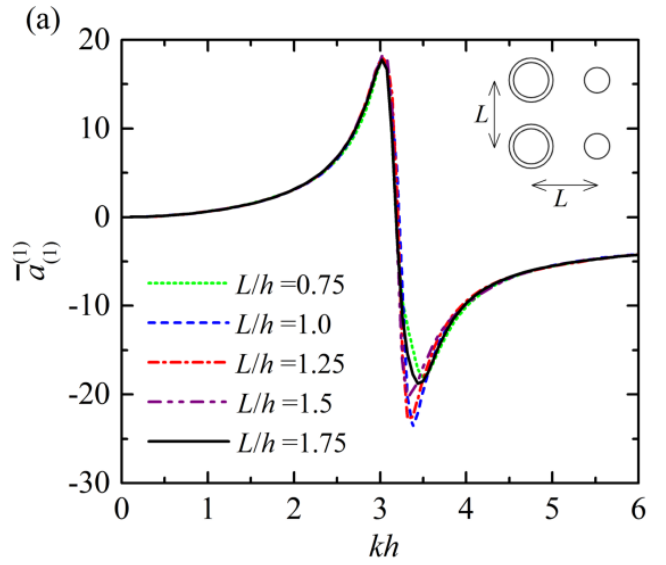

(c)

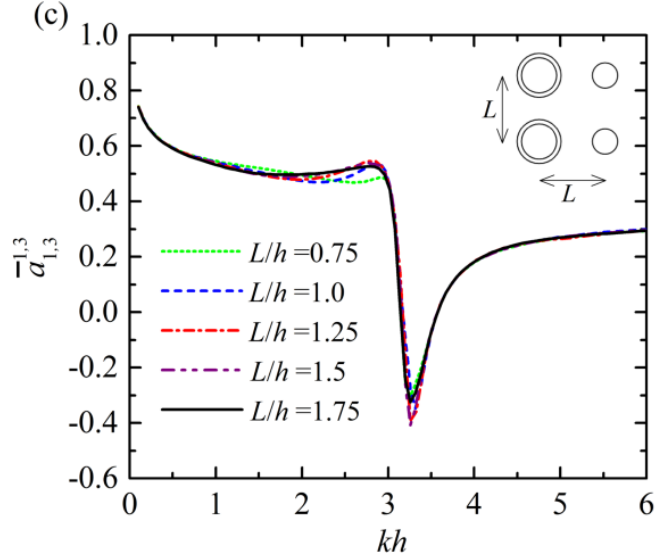

(e)

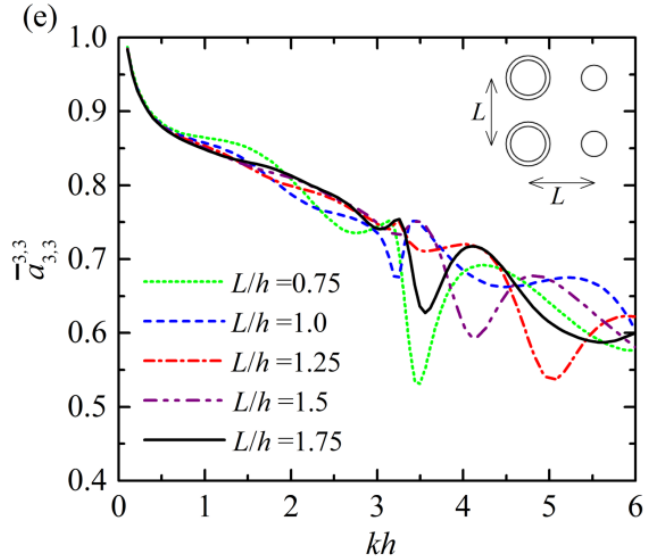

(b)

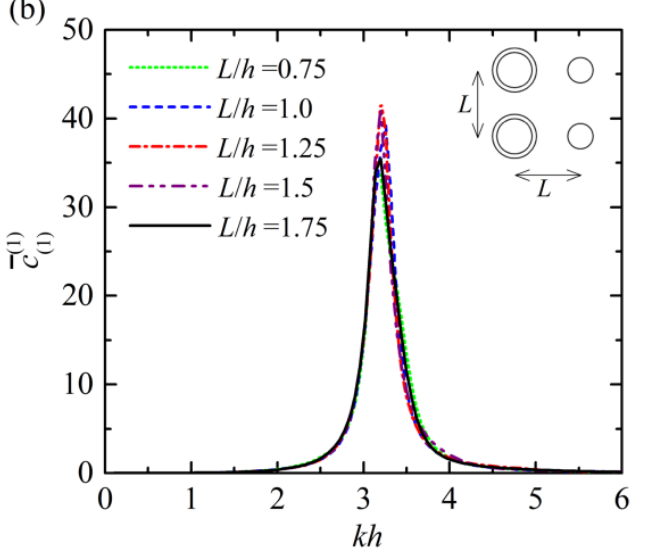

(d)

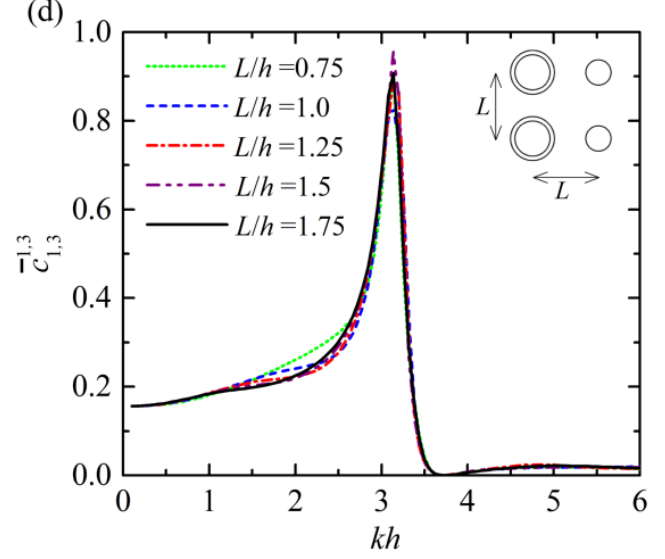

(f)

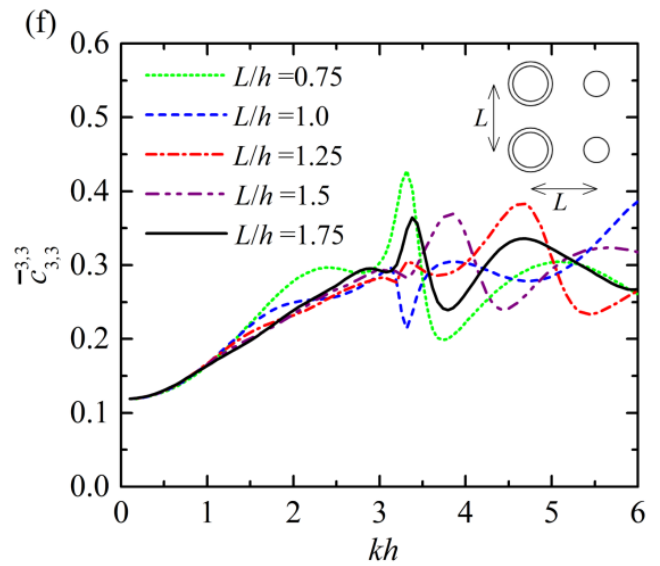

Fig. 14. Hydrodynamic coefficients of the WECs in the hybrid wave farm \#H1 versus $k h$ for different $L / h$ : (a) $\bar{a}_{(1)}^{(1)}$; (b) $\bar{c}_{(1)}^{(1)}$; (c) $\bar{a}_{1,3}^{1,3}$;(d) $\bar{c}_{1,3}^{1,3}$; (e) $\bar{a}_{3,3}^{3,3}$;(f) $\bar{c}_{3,3}^{3,3}$. 
(a)

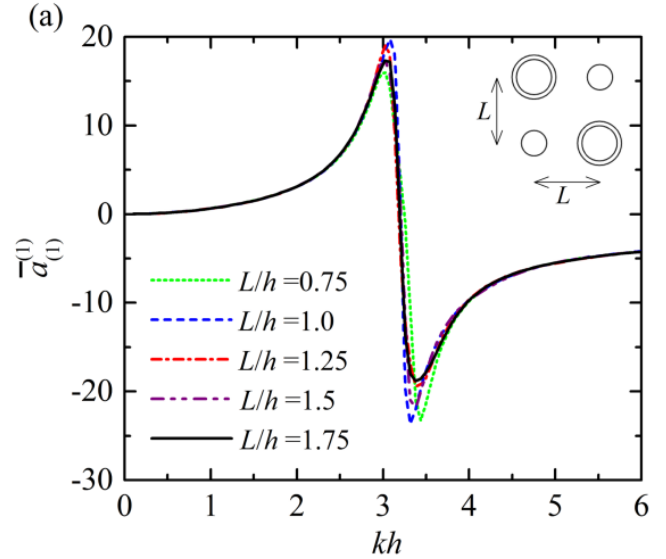

(c)

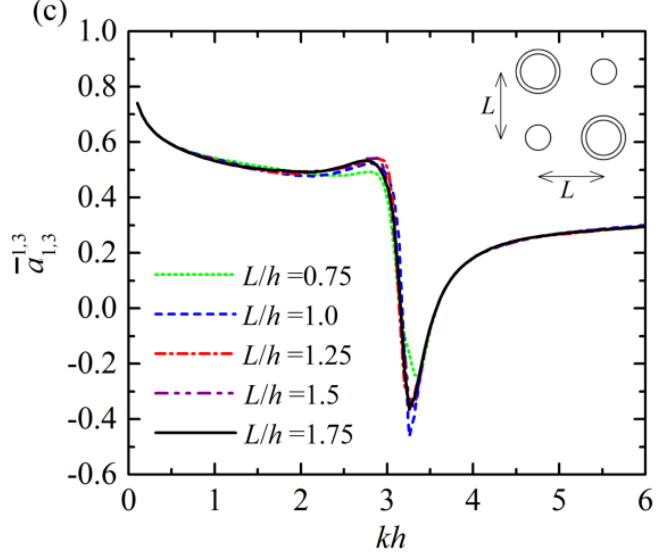

(e)

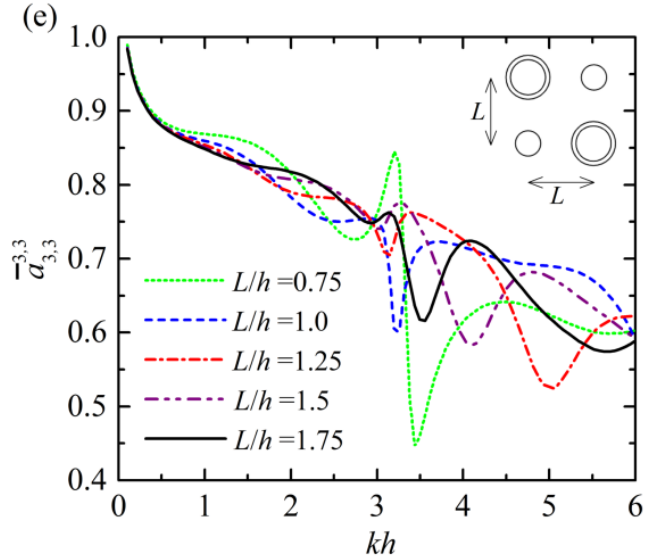

(b)

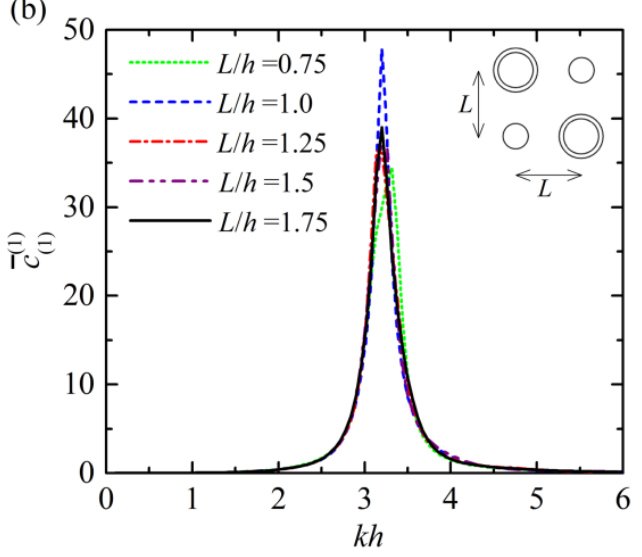

(d)

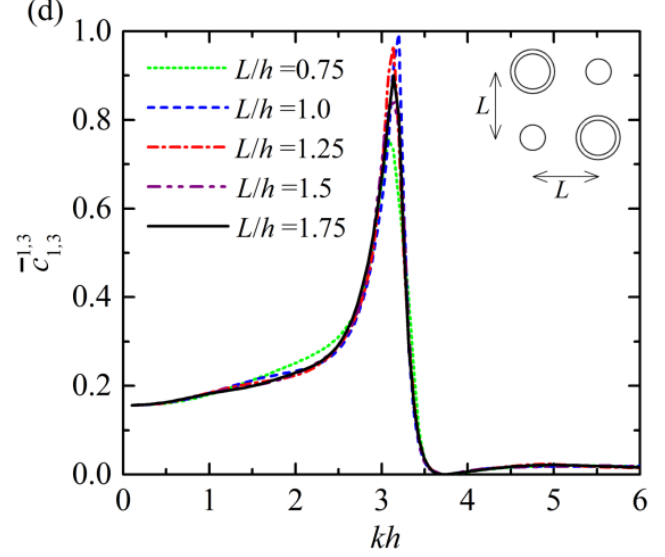

(f) 0.7

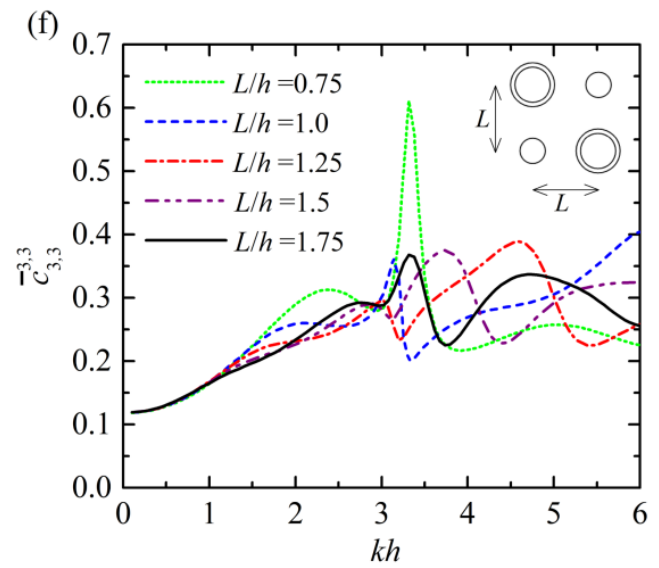

Fig. 15. Hydrodynamic coefficients of the WECs in the hybrid wave farm \#H2 versus $k h$ for different $L / h$ : (a) $\bar{a}_{(1)}^{(1)}$; (b) $\bar{c}_{(1)}^{(1)}$; (c) $\bar{a}_{1,3}^{1,3}$;(d) $\bar{c}_{1,3}^{1,3}$; (e) $\bar{a}_{3,3}^{3,3}$;(f) $\bar{c}_{3,3}^{3,3}$.

For $\bar{a}_{1,3}^{1,3}$ and $\bar{c}_{1,3}^{1,3}$, as shown in Figs. $14 \mathrm{c}, 14 \mathrm{~d}$ and $15 \mathrm{c}, 15 \mathrm{~d}$, their spikes occur approximately at $k h=3.2$, as with $\bar{c}_{(1)}^{(1)}$ and $\bar{a}_{(1)}^{(1)}$. The height of the spike in $\bar{c}_{1,3}^{1,3}$ and the total extent of $\bar{a}_{1,3}^{1,3}$ are also found to be nearly equal. Apart from the range $3.0<k h<4.0$, a limited influence of $L / h$ may also be observed for $1.5<k h<3.0$. Regardless of \#H1 and \#H2, note $\bar{c}_{1,3}^{1,3}$ for 
$L / h=0.75$ is obviously larger than those for the other values of $L / h$ at $1.5<k h<2.5$. In Figs. $14 \mathrm{e}, 14 \mathrm{f}$ and $15 \mathrm{e}, 15 \mathrm{f}$, both $\bar{a}_{3,3}^{3,3}$ and $\bar{c}_{3,3}^{3,3}$ are found significantly dependent on $L / h$ for most wave conditions, which is very different compared the influence of $L / h$ on $\bar{a}_{(1)}^{(1)}, \bar{c}_{(1)}^{(1)}, \bar{a}_{1,3}^{1,3}$ and $\bar{c}_{1,3}^{1,3}$. This can be explained from the view of submerge depth difference between OWC and PA. The submerged depth of OWC is $0.2 h$, one order of magnitude larger than that of PA $(d=0.024 h)$. Therefore, the radiated waves induced by the heave motion of the OWC chamber (or air pressure fluctuation) are generally much weaker than those induced by the PA oscillating in heave mode with the same amplitude. Consequently, the diffraction of those small radiated waves caused by OWC chamber heave motion (or air pressure fluctuation) within the hybrid wave farm is negligible for most wave conditions. As a result, effect of $L / h$ is mainly found on $\bar{a}_{3,3}^{3,3}$ and $\bar{c}_{3,3}^{3,3}$ rather than on $\bar{a}_{(1)}^{(1)}, \bar{c}_{(1)}^{(1)}, \bar{a}_{1,3}^{1,3}$ and $\bar{c}_{1,3}^{1,3}$.

\section{Conclusions}

In this paper, a hybrid wave farm consisting of two types of WEC, OWCs and PAs, was proposed for the first time, and its interaction with the wave field was investigated by means of a semi-analytical model based on linear potential flow theory. The OWCs and PAs are truncated cylinders with and without moonpools, respectively, and have different outer radius, inner radius, draft and mass. It was assumed that the velocity potential at any fluid point can be written as a sum of wave incident potential, diffracted potential and radiated potentials due to the oscillation of the OWCs/PAs and the pressure fluctuation of the air inside the OWC chambers. The entire water domain was divided into the subdomains beneath the OWCs/PAs, those enclosed by the OWC chambers extended to the seabed, and the rest outside OWCs/PAs. By means of the method of separation of variables, the diffracted and radiated spatial velocity potentials in the different subdomains were described by a series of Fourier functions with unknown coefficients, which were determined by adopting the continuity conditions of pressure and velocity between adjacent subdomains, together with the eigen-function matching method. Once the diffraction and radiation problems had been solved, the wave excitation volume flux/forces were directly evaluated with incident and diffracted potentials. The hydrodynamic coefficients were calculated from the radiated potentials directly, and some of them were also obtained indirectly by integrating the wave excitation volume flux/forces over the entire range of incident wave directions based on the Haskind relation.

For validation, this semi-analytical model was used to solve the wave diffraction and radiation problems of a hybrid wave farm case consisting of two OWCs and two PAs with arbitrarily specified scales. Excellent agreement is obtained with the numerical results from a BEM software.

Upon validation, the model was applied to investigate the hydrodynamic performance of two configurations of a hybrid wave farm consisting of two OWCs and two PAs, the scales of which are selected from previous researchers Nader (2013), Konispoliatis and Mavrakos (2016) and Göteman (2017), respectively, i.e., PA: $R_{n} / h=0.14, d_{n} / h=0.024 ;$ OWC: $R_{n} / h=0.25, R_{\mathrm{i}, h} / h=0.2$, $d_{n} / h=0.2$. The two configurations are: \#H1, with the two WECs of the same type (either OWC or $\mathrm{PA}$ ) on the same row, and $\# \mathrm{H} 2$, with one OWC and one PA on each row. We examined how the 
incident wave direction $(\beta)$ and spacing, i.e., length of the square side line $(L)$, affect the wave excitation volume flux/forces and hydrodynamic coefficients.

The following conclusions may be drawn:

(i) For configuration $\# \mathrm{H} 2$ and $L / h=0.75$, when the incident waves propagate along the diagonal line of the two OWCs, the excitation volume flux and the heave excitation force acting on the lee side OWC is larger than those acting on the wind side OWC for $2.5<k h<3.5$. This could be due to the waves diffracted from the two PAs toward the lee side OWC.

(ii) For $k h=3.3$ and $L / h=0.75$, the heave wave excitation forces acting on each OWC/PA and the volume flux of each OWC in configuration $\# \mathrm{H} 1$ are all at a rather large level when incident waves propagate from the side of PAs, i.e., $\beta=\pi$, hence configuration $\# \mathrm{H} 1$ is expected to absorb more wave power under that incident wave direction. For \#H2 with $k h=3.3$ and $L / h=0.75$, $\beta=0.25 \pi$ is a better choice.

(iii) For configuration $\# \mathrm{H} 1$ under waves with $\beta=\pi$, as $L / h$ increases from 0.75 to 1.75 , the peak values of $\bar{F}_{\mathrm{e}}^{(1)}-k h$ first increase and then decrease, whereas the $k h$ corresponding to the peak value decreases all the time. A similar trend of the peak in terms of both peak amplitude and the corresponding $k h$ with $L / h$ also applies to $\bar{F}_{\mathrm{e}}^{1,3}$.

(iv) For a hybrid wave farm with the submerged depth of OWC much larger than that of PA, changing $L / h$ has a greater influence on $\bar{a}_{3,3}^{3,3}$ and $\bar{c}_{3,3}^{3,3}$ compared with those on $\bar{a}_{(1)}^{(1)}, \bar{c}_{(1)}^{(1)}, \bar{a}_{1,3}^{1,3}$ and $\bar{c}_{1,3}^{1,3}$.

In sum, the semi-analytical model proposed in this paper was proven to be accurate and effective for assessing the wave excitation volume flux/forces and hydrodynamic coefficients of OWCs and PAs in a hybrid wave farm, and provides a more efficient method compared with a numerical model. The development of this model is the first step toward analytically evaluating the motion/pressure response and performance of a hybrid wave farm in capturing wave power from ocean waves, which is the topic of our next paper. Note energy dissipation might be induced around the sharp corners, while in this paper, such effect was assumed to be negligible.

\section{Acknowledgements}

The research was supported by China Postdoctoral Science Foundation (Grant No. 2016M601041, 2017T100085). Intelligent Community Energy (ICE), INTERREG V FCE, European Commission (Contract No. 5025).

Appendix A. Derivation process of the formulas and calculation for the unknown coefficients of both diffracted and radiated potentials

Substitute the diffracted spatial potentials in Eqs. (12) (22) and wave incident potential given in Eq.(2b) into Eqs. (23) (24), then after multiplying both sides by $\mathrm{e}^{-\mathrm{i} \tau \theta_{n}} \cos \left[\beta_{n, \zeta}(z+h)\right]$ and integrating for $\theta_{n} \in[0,2 \pi]$ and $z \in\left[-h,-d_{n}\right]$, we get: 


$$
\begin{aligned}
& -A_{\tau, \zeta}^{\mathrm{D}, n}-\xi_{n, M} C_{\tau, \zeta}^{\mathrm{D}, n}+2 \sum_{l=0}^{\infty} B_{\tau, l}^{\mathrm{D}, n} L_{l, \zeta}^{(n)}+2 \sum_{\substack{j=1 \\
j \neq n}}^{M+N} \sum_{m=-\infty}^{\infty} \sum_{l=0}^{\infty} B_{m, l}^{\mathrm{D}, j} T_{m, \tau, l}^{n, j} L_{l, \zeta}^{(n)} \\
& =\frac{2 \mathrm{i} g A}{\omega} \mathrm{i}^{\tau} J_{\tau}\left(k_{0} R_{n}\right) L_{0, \zeta}^{(n)} \mathrm{e}^{\mathrm{i} k_{0}\left(x_{n} \cos \beta+y_{n} \sin \beta\right)} \mathrm{e}^{-\mathrm{i} \tau \beta}, \quad n=1,2, \cdots, M+N
\end{aligned}
$$

$$
-a_{\tau, \zeta}^{(n)} A_{\tau, \zeta}^{\mathrm{D}, n}-b_{\tau, \zeta}^{(n)} C_{\tau, \zeta}^{\mathrm{D}, n}+2 \sum_{l=0}^{\infty} D_{\tau, l}^{\mathrm{D}, M+N+n} L_{l, \zeta}^{(n)}
$$

$$
=\frac{2 \mathrm{i} g A}{\omega} \mathrm{i}^{\tau} J_{\tau}\left(k_{0} R_{\mathrm{i}, n}\right) L_{0, \zeta}^{(n)} \mathrm{e}^{\mathrm{i} k_{0}\left(x_{n} \cos \beta+y_{n} \sin \beta\right)} \mathrm{e}^{-\mathrm{i} \tau \beta}, \quad n=1,2, \cdots, M
$$

3

where

4

$$
\begin{aligned}
& \xi_{n, M}=\left\{\begin{array}{ll}
1, & n \leq M \\
0, & n>M
\end{array},\right. \\
& L_{l, \varsigma}^{(n)}=\frac{1}{h-d_{n}} \int_{-h}^{-d_{n}} \frac{Z_{l}(z) \cos \left[\beta_{n, \varsigma}(z+h)\right]}{Z_{l}(0)} \mathrm{d} z \\
& =\left\{\begin{array}{ll}
\frac{(-1)^{\varsigma}\left(h-d_{n}\right) k_{0} \sinh \left[k_{0}\left(h-d_{n}\right)\right]}{\left[\left(h-d_{n}\right)^{2} k_{0}^{2}+\varsigma^{2} \pi^{2}\right] \cosh \left(k_{0} h\right)}, & l=0 ; \varsigma=0,1,2 \cdots \\
\frac{(-1)^{\varsigma}\left(h-d_{n}\right) k_{l} \sin \left[k_{l}\left(h-d_{n}\right)\right]}{\left[\left(h-d_{n}\right)^{2} k_{l}^{2}-\varsigma^{2} \pi^{2}\right] \cos \left(k_{l} h\right)}, & l=1,2,3 \cdots ; \varsigma=0,1,2 \cdots
\end{array},\right. \\
& T_{m, \tau, l}^{n, j}=\left\{\begin{array}{ll}
\frac{(-1)^{\tau} H_{m-\tau}\left(k_{0} R_{n j}\right) J_{\tau}\left(k_{0} R_{n}\right) \mathrm{e}^{\mathrm{i}\left(m \alpha_{j n}-\tau \alpha_{n j}\right)}}{H_{m}\left(k_{0} R_{j}\right)}, & l=0 \\
\frac{K_{m-\tau}\left(k_{l} R_{n j}\right) I_{\tau}\left(k_{l} R_{n}\right) \mathrm{e}^{\mathrm{i}\left(m \alpha_{j n}-\tau \alpha_{n j}\right)}}{K_{m}\left(k_{l} R_{j}\right)}, & l=1,2,3 \cdots
\end{array},\right. \\
& a_{\tau, \zeta}^{(n)}=\left\{\begin{array}{ll}
\left(\frac{R_{\mathrm{i}, n}}{R_{n}}\right)^{|\tau|}, & \zeta=0 \\
\frac{I_{\tau}\left(\beta_{n, \zeta} R_{\mathrm{i}, n}\right)}{I_{\tau}\left(\beta_{n, \zeta} R_{n}\right)}, & \zeta \neq 0
\end{array} ; b_{\tau, \zeta}^{(n)}= \begin{cases}1+\ln \left(\frac{R_{\mathrm{i}, n}}{R_{n}}\right), & \zeta=0 ; \tau=0 \\
\left(\frac{R_{\mathrm{i}, n}}{R_{n}}\right)^{-|\tau|}, & \zeta=0 ; \tau \neq 0 . \\
\frac{K_{\tau}\left(\beta_{n, \zeta} R_{\mathrm{i}, n}\right)}{K_{\tau}\left(\beta_{n, \zeta} R_{n}\right)}, & \zeta \neq 0\end{cases} \right.
\end{aligned}
$$

8 Similarly, substitute the diffracted spatial potentials in Eqs. (12) (22) and wave incident 9 potential given in Eq.(2b) into Eqs. (25) (26), after multiplying both sides by $\mathrm{e}^{-\mathrm{i} \tau \theta_{n}} Z_{\varsigma}(z)$ and integrating for $\theta_{n} \in[0,2 \pi]$ and $z \in[-h, 0]$, we have: 
1

$-\sum_{l=0}^{\infty} A_{\tau, l}^{\mathrm{D}, n} \Lambda_{\tau, l}^{(n)} Z_{\zeta}(0) L_{\zeta, l}^{(n)}-\xi_{n, M} \sum_{l=0}^{\infty} C_{\tau, l}^{\mathrm{D}, n} X_{\tau, l}^{(n)} Z_{\zeta}(0) L_{\zeta, l}^{(n)}+B_{\tau, \zeta}^{\mathrm{D}, n} \Pi_{\tau, \zeta}^{(n)}+\sum_{\substack{j=1 \\ j \neq n}}^{M+N} \sum_{m=-\infty}^{\infty} B_{m, \zeta}^{\mathrm{D}, j} T_{m, \tau, \zeta}^{\prime n, j}$

$$
=\delta_{\zeta, 0} \frac{\mathrm{i}^{\tau+1} \mathrm{e}^{\mathrm{i} k_{0}\left(x_{n} \cos \beta+y_{n} \sin \beta\right)} \mathrm{e}^{-\mathrm{i} \tau \beta} g A k_{0} J_{\tau}^{\prime}\left(k_{0} R_{n}\right)}{\omega Z_{0}(0)}, \quad n=1,2, \cdots, M+N
$$

2

3

$$
\begin{aligned}
& -\sum_{l=0}^{\infty}{a_{\tau, l}^{\prime(n)}}^{(n)} A_{\tau, l}^{\mathrm{D}, n} \Lambda_{\tau, l}^{(n)} Z_{\zeta}(0) L_{\zeta, l}^{(n)}-\sum_{l=0}^{\infty} b_{\tau, l}^{\prime(n)} C_{\tau, l}^{\mathrm{D}, n} X_{\tau, l}^{(n)} Z_{\zeta}(0) L_{\zeta, l}^{(n)}+D_{\tau, \zeta}^{\mathrm{D}, M+N+n} \Pi_{\tau, \zeta}^{(n)} \\
& =\delta_{\zeta, 0} \frac{\mathrm{i}^{\tau+1} \mathrm{e}^{\mathrm{i} k_{0}\left(x_{n} \cos \beta+y_{n} \sin \beta\right)} \mathrm{e}^{-\mathrm{i} \tau \beta} g A k_{0} J_{\tau}^{\prime}\left(k_{0} R_{\mathrm{i}, n}\right)}{\omega Z_{0}(0)}, \quad n=1,2, \cdots, M
\end{aligned}
$$

4 where

5

$$
\Lambda_{\tau, l}^{(n)}= \begin{cases}\frac{|\tau|\left(h-d_{n}\right)}{2 R_{n} h}, & l=0 \\ \frac{\beta_{n, l} I_{\tau}^{\prime}\left(\beta_{n, l} R_{n}\right)\left(h-d_{n}\right)}{I_{\tau}\left(\beta_{n, l} R_{n}\right) h}, & l=1,2,3 \cdots\end{cases}
$$

6

$$
X_{\tau, l}^{(n)}=\left\{\begin{array}{l}
\frac{h-d_{n}}{2 R_{n} h}, \quad l=0, \tau=0 \\
\frac{-|\tau|\left(h-d_{n}\right)}{2 R_{n} h}, \quad l=0, \tau \neq 0 \\
\frac{\beta_{n, l} K_{\tau}^{\prime}\left(\beta_{n, l} R_{n}\right)\left(h-d_{n}\right)}{K_{\tau}\left(\beta_{n, l} R_{n}\right) h}, \quad l=1,2,3 \cdots
\end{array},\right.
$$

$7 \quad \Pi_{\tau, l}^{(n)}=\left\{\begin{array}{l}\frac{k_{0} H_{\tau}^{\prime}\left(k_{0} R_{n}\right)}{H_{\tau}\left(k_{0} R_{n}\right) Z_{0}(0)}, l=0 \\ \frac{k_{l} K_{\tau}^{\prime}\left(k_{l} R_{n}\right)}{K_{\tau}\left(k_{l} R_{n}\right) Z_{l}(0)}, l=1,2,3 \cdots\end{array} \quad ; \quad \Pi_{\tau, l}^{(n)}=\left\{\begin{array}{l}\frac{k_{0} J_{\tau}^{\prime}\left(k_{0} R_{\mathrm{i}, n}\right)}{J_{\tau}\left(k_{0} R_{\mathrm{i}, n}\right) Z_{0}(0)}, l=0 \\ \frac{k_{l} I_{\tau}^{\prime}\left(k_{l} R_{\mathrm{i}, n}\right)}{I_{\tau}\left(k_{l} R_{\mathrm{i}, n}\right) Z_{l}(0)}, l=1,2,3 \cdots\end{array}\right.\right.$

8

9

$$
T_{m, \tau, l}^{n, j}=\left\{\begin{array}{ll}
\frac{(-1)^{\tau} k_{0} J_{\tau}^{\prime}\left(k_{0} R_{n}\right) H_{m-\tau}\left(k_{0} R_{n j}\right) \mathrm{e}^{\mathrm{i}\left(m \alpha_{j n}-\tau \alpha_{n j}\right)}}{H_{m}\left(k_{0} R_{j}\right) Z_{0}(0)}, & l=0 \\
\frac{k_{l} I_{\tau}^{\prime}\left(k_{l} R_{n}\right) K_{m-\tau}\left(k_{l} R_{n j}\right) \mathrm{e}^{\mathrm{i}\left(m \alpha_{j n}-\tau \alpha_{m i j}\right)}}{K_{m}\left(k_{l} R_{j}\right) Z_{l}(0)}, & l=1,2,3 \cdots
\end{array},\right.
$$




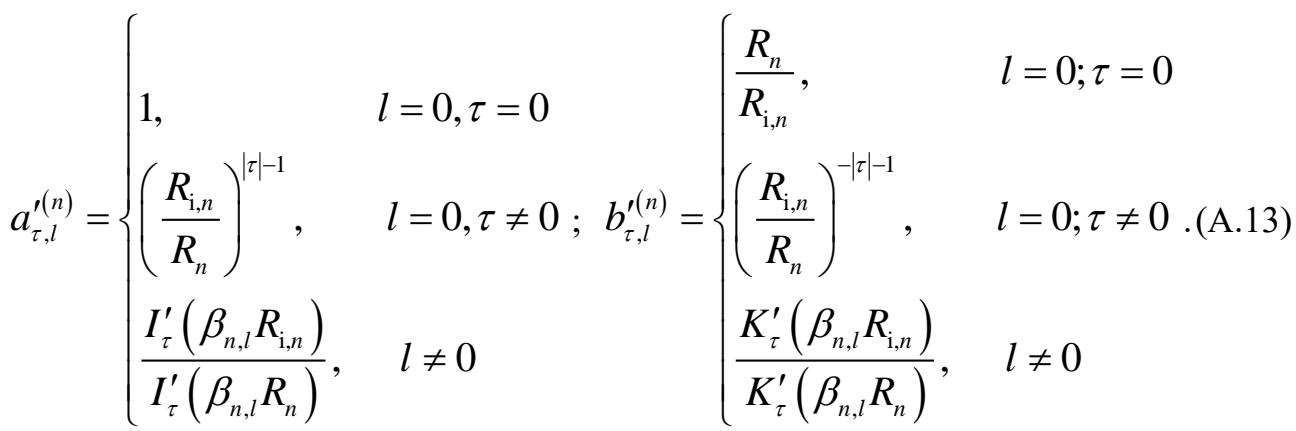

$2(2 M+N)\left(2 m_{0}+1\right)\left(l_{0}+1\right)$ complex linear equations with an equal number of unknown coefficients can be obtained from Eqs.(A1), (A2), (A7) and (A8) after truncation of $\mathrm{e}^{\mathrm{i} m \theta}$ $\left(m=-m_{0}, \ldots, 0, \ldots, m_{0}\right), Z_{l}(\mathrm{z})$ and $\cos \left[\beta_{n, l}(z+h)\right]\left(l=0,1,2, \ldots L_{0}\right)$ functions. Therefore, the unknown coefficients can be easily calculated by solving the complex $2(2 M+N)\left(2 m_{0}+1\right)\left(l_{0}+1\right)$ order linear matrix equation.

For the radiated spatial potentials due to float oscillation of all the floats and air pressure oscillation inside the OWC chambers, similar expressions can also be derived. Derivation shows that the diffracted and radiated spatial potentials share the same $2(2 M+N)\left(2 m_{0}+1\right)\left(l_{0}+1\right)$ order linear complex coefficient matrix, hence wave diffraction and wave radiation problems can be solved simultaneously.

\section{References}

Abanades, J., Greaves, D., Iglesias, G., 2014. Coastal defence using wave farms: The role of farm-to-coast distance. Renewable Energy, 75, 572-582.

Abanades, J., Greaves, D., Iglesias, G., 2015. Wave farm impact on beach modal state. Marine Geology. 361, 126-135.

Abramowitz, M., Stegun, I.A., 1964. Handbook of Mathematical Functions. Government Printing Office, Washingtion, DC.

ANSYS AQWA, 2011. Release. vol. 14. ANSYS-Inc., Pennsylvania, USA.

Astariz, S., Iglesias, G., 2015a. Enhancing wave energy competitiveness through co-located wind and wave energy farms. A review on the shadow effect. Energies. 8, 7344-7366.

Astariz, S., Iglesias, G., 2015b. Accessibility for operation and maintenance tasks in co-located wind and wave energy farms with non-uniformly distributed arrays. Energy Conversion and Management. 106, 1219-1229.

Astariz, S., Iglesias, G., 2016. Selecting optimum locations for co-located wave and wind energy farms. Part I: The Co-Location Feasibility index. Energy Conversion and Management. 122, 589-598.

Astariz, S., Perez-Collazo, C., Abanades, J., Iglesias, I., 2015. Towards the optimal design of a co-located wind-wave farm. Energy. 84, 15-24.

Babarit, A., 2010. Impact of long separating distances on the energy production of two interacting wave energy converters. Ocean Engineering. 37, 718-729.

Bacelli, G., Ringwood, J., 2013. Constrained control of arrays of wave energy devices. International Journal of Marine Energy. (3-4), e53-e69.

Borgarino, B., Babarit, A., Ferrant, P., 2012. Impact of wave interactions effects on energy absorption in large arrays of wave energy converters. Ocean Engineering. 41, 79-88. 
Carballo, R., Iglesias, G., 2013. Wave farm impact based on realistic wave-WEC interaction. Energy. 51, 216-229.

Carballo, R., Sánchez, M., Ramos, V, Fraguela, J.A., Iglesias, G., 2015a. The intra-annual variability in the performance of wave energy converters: a comparative study in N Galicia (Spain). Energy. 82, 138-146.

Carballo, R., Sánchez, M., Ramos, V., Fraguela, J.A., Iglesias, G., 2015b. Intra-annual wave resource characterization for energy exploitation: a new decision-aid tool. Energy Conversion and Management. 93, 1-8.

Child, B.F.M., Venugopal, V., 2010. Optimal configurations of wave energy device arrays. Ocean Engineering. 37(16), 1402-1417.

Cruz, J., Sykes, R., Siddorn, P., Taylor, R.R., 2010. Estimating the loads and energy yield of arrays of wave energy converters under realistic seas. IET Renewable Power Generation. 4(6), 488-497.

de Andres, A.D., Guanche, R., Meneses, L., Vidal, C., Losada, I.J., 2014. Factors that influence array layout on wave energy farms. Ocean Engineering. 82, 32-41.

De Backer, G., Vantorre, M., Beels, C., De Rouck, J., Frigaard, P., 2010. Power absorption by closely spaced point absorbers in constrained conditions. IET Renewable Power Generation. 4(6), 579-591.

Devolder, B., Stratigaki, V., Troch, P., Rauwoens, P., 2018. CFD simulations of floating point absorber wave energy converter arrays subjected to regular waves. Energies. 11(3), 641-663.

Drew, B., Plummer, A.R., Sahinkaya, M.N., 2009. A review of wave energy converter technology. Proceedings of the Institution of Mechanical Engineers, Part A: Journal of Power and Energy. 223, 887-902.

Falcão, A.F.O., 2010. Wave energy utilization: A review of the technologies. Renewable and Sustainable Energy Reviews. 14, 899-918.

Falnes, J., 2002. Ocean waves and oscillating systems. Cambridge University Press, Cambridge, UK.

Finnegan, W., Meere, M., Goggins, J., 2013. The wave excitation forces on a truncated vertical cylinder in water of infinite depth. Journal of Fluids and Structures. 40, 201-213.

Flavià, F.F., Babarit, A., Clément, A.H., 2017. On the numerical modeling and optimization of a bottom-referenced heave-buoy array of wave energy converters. International Journal of Marine Energy. 19, 1-15.

Folley, M., Whittaker, T.J.T., 2009. The effect of sub-optimal control and the spectral wave climate on the performance of wave energy converter arrays. Applied Ocean Research. 31, 260-266.

Fusco, F., Nolan, G., Ringwood, J.V., 2010. Variability reduction through optimal combination of wind/wave resources - An Irish case study. Energy. 35(1), 314-325.

Garnaud, X., Mei, C.C., 2009. Wave-power extraction by a compact array of buoys. Journal of Fluid Mechanics, 635, 389-413.

Göteman, M., 2017. Wave energy parks with point-absorbers of different dimensions. Journal of Fluids and Structures. 74, 142-157.

Göteman, M., Engström, J., Eriksson, M., Isberg, J., Leijon, M., 2014. Methods of reducing power fluctuations in wave energy parks. Journal of Renewable and Sustainable Energy. 6, 043103(2014). 
Haller, M., Porter, A., Lenee-Bluhm, P., Rhinefrank, K., Hammagren, E., Ozkan-Haller, T., Newborn, D., 2011. Laboratory observations of waves in the vicinity of WEC-arrays. Proceedings of the $9^{\text {th }}$ European Wave and Tidal Energy Conference. September 4-11, Southampton, UK.

Iglesias, G., Carballo, R., 2014. Wave farm impact: the role of farm-to-coast distance. Renewable Energy. 69, 375-385.

Iuppa, C., Cavallaro, L., Foti, E., Vicinanza, D., 2015. Potential wave energy production by different wave energy converters around Sicily. Journal of Renewable and Sustainable Energy. 7, 061701 (2015) https://doi.org/10.1063/1.4936397

Khojasteh, D., Khojasteh, D., Kamali, R., Beyene, A., Iglesias, G., 2018. Assessment of Renewable Energy Resources in Iran; with a Focus on Wave and Tidal Energy. Renewable and Sustainable Energy Reviews, 81 (2), 2992-3005.

Konispoliatis, D.N., Mavrakos, S.A., 2016. Hydrodynamic analysis of an array of interacting free-floating oscillating water column (OWC's) devices. Ocean Engineering. 111, 179-197.

Linton, C.M., Evans, D.V., 1993. Hydrodynamic characteristics of bodies in channels. Journal Fluid Mechanics. 252, 647-666.

López, I., Andreu, J., Ceballos, S., Martinez de Alegra, I., Kortabarria, I., 2013. Review of wave energy technologies and the necessary power-equipment. Renewable and Sustainable Energy Reviews. 27, 413-434.

López, I., Iglesias, G., 2014. Efficiency of OWC wave energy converters: a virtual laboratory. Applied Ocean Research. 44, 63-70.

López, I., Pereiras, B., Castro, F., Iglesias, G., 2015b. Performance of OWC wave energy converters: influence of turbine damping and tidal variability. International Journal of Energy Research. 39, 472-483.

López, M., Veigas, M., Iglesias, G., 2015a. On the wave energy resource of Peru. Energy Conversion and Management. 90, 34-40.

López-Ruiz, A., Bergillos, R.J., Raffo-Caballero, J.M., Ortega-Sánchez, M., 2018. Towards an optimum design of wave energy converter arrays through an integrated approach of life cycle performance and operational capacity. Applied Energy. 209, 20-32.

Mustapa, M.A., Yaakob, O.B., Ahmed, Y.M., Rheem, C.K., Koh, K.K., Adnan, F.A., 2017. Wave energy device and breakwater integration: A review. Renewable and Sustainable Energy Reviews. 77, 43-58.

Nader, J.R., 2013. Interaction of ocean waves with oscillating water column wave energy convertors. University of Wollongong, Australia, $\mathrm{PhD}$.

Nokob, M.H., Yeung, R.W., 2015. Diffraction and radiation loads on open cylinders of thin and arbitrary shapes. Journal Fluid Mechanics. 772, 649-677.

Penalba, M., Touzón, I., Lopez-Mendia,J., Nava, V., 2017. A numerical study on the hydrodynamic impact of device slenderness and array size in wave energy farms in realistic wave climates. Ocean Engineering. 142, 224-232.

Pereiras, B., López, I., Castro, F., Iglesias, G., 2015. Non-dimensional analysis for matching an impulse turbine to an OWC (oscillating water column) with an optimum energy transfer. Energy. 87, 481-489.

Rahm, M., Svensson, O., Boström, C., Waters, R., Leijon, M., 2012. Experimental results from the operation of aggregated wave energy converters. IET Renewable Power Generation. 6(3), 
149-160.

Renzi, E., Abdolali, A., Bellotti, G., Dias, F., 2014. Wave-power absorption from a finite array of oscillating wave surge converters. Renewable Energy. 63, 55-68.

Renzi, E., Dias, F., 2013a. Relations for a periodic array of flap-type wave energy converters. Applied Ocean Research. 39, 31-39.

Renzi, E., Dias, F., 2013b. Hydrodynamics of the oscillating wave surge converter in the open ocean. European Journal of Mechanics-B/Fluids. 41, 1-10.

Ross, D., 1995. Power from sea waves. Oxford: Oxford University Press.

Sarkar, D., Renzi, E., Dias, F., 2014a. Wave farm modelling of oscillating wave surge converters. Proceedings of the Royal Society A. 470: 20140118.

Sarkar, D., Renzi, E., Dias, F., 2014b. Interactions between an oscillating wave surge converter and a heaving wave energy converter. Journal of Ocean and Wind Energy. 1(3), 135-142.

Sarkar, D., Renzi, E., Dias, F., 2015. Effect of a straight coast on the hydrodynamics and performance of the Oscillating Wave Surge Converter. Ocean Engineering. 105, 25-32.

Sheng, W., Alcorn, R., Lewis, A., 2014a. Assessment of primary energy conversions of oscillating water columns. I. Hydrodynamic analysis. Journal of Renewable and Sustainable Energy. 6, 053113 (2014). https://doi.org/10.1063/1.4896850

Sheng, W., Alcorn, R., Lewis, A., 2014b. Assessment of primary energy conversions of oscillating water columns. II. Power take-off and validations. Journal of Renewable and Sustainable Energy. 6, 053114 (2014). https://doi.org/10.1063/1.4896851

Sheng, W., Alcorn, R., Lewis, T., 2015. On improving wave energy conversion, part I: Optimal and control technologies. Renewable Energy. 75,922-934.

Siddorn, P., Eatock Taylor, R., 2008. Diffraction and independent radiation by an array of floating cylinders. Ocean Engineering. 35, 1289-1303.

Thomas, S., Weller, S., Stallard, T., 2008. Float response within an array: numerical and experimental comparison. Proceedings of the 2nd International Conference on Ocean Energy, 15th-17th October 2008, Brest, France.

Venugopal, V., Smith, G.H., 2007. Wave climate investigation for an array of wave power devices. Proceedings of the $7^{\text {th }}$ European Wave and Tidal Energy Conference. September 11-14, Porto, Portugal.

Viviano, A., Naty, S., Foti, E., Bruce, T., Allsop, W., Vicinanza, D., 2016. Large-scale experiments on the behaviour of generalised Oscillating Water Column under random waves. Renewable Energy. 99, 875-887.

Weller, S.D., Stallard, T.J., Stansby, P.K., 2010. Experimental measurements of irregular wave interaction factors in closely spaced arrays. IET Renewable Power Generation. 4(6), 628-637.

Wu, J., Yao, Y., Zhou, L., et al., 2017. Performance analysis of solo Duck wave energy converter arrays under motion constraints. Energy. 139, 155-169.

Zheng, S., Zhang Y., 2015. Wave diffraction from a truncated cylinder in front of a vertical wall. Ocean Engineering. 104, 329-343.

Zheng, S., Zhang Y., 2016. Wave radiation from a truncated cylinder in front of a vertical wall. Ocean Engineering. 111, 602-614.

Zheng, S., Zhang Y., 2017a. Analysis for wave power capture capacity of two interconnected floats in regular waves. Journal of Fluids and Structures. 75, 158-173.

Zheng, S., Zhang, Y., 2017b. Analytical study on hydrodynamic performance of a raft-type wave 
1 power device. Journal of Marine Science and Technology. 22(4), 620-632.

2 Zheng, S, Zhang, Y., Sheng, W., 2016a. Maximum theoretical power absorption of connected

3 floating bodies under motion constraints. Applied Ocean Research. 58, 95-103.

4 Zheng, S., Zhang, Y., Sheng, W., 2016b. Maximum wave energy conversion by two 5 interconnected floaters. Journal of Energy Resources Technology. 138(3), 032004.

6 Zheng, S., Zhang, Y., Zhang, Y., Sheng, W., 2015. Numerical study on the dynamics of a two-raft wave energy conversion device. Journal of Fluids and Structures. 58, 271-290. 